\title{
1 THEORETISCHE UND KONZEPTIONELLE GRUNDLAGEN
}

\subsection{Einleitung}

Die selbständige Erwerbstätigkeit von Immigrant/inn/en generell leistet einen immer wichtiger werdenden Beitrag zur Entwicklung in vielen Wirtschaftssektoren. Sie ist zugleich ein wichtiger Motor der strukturellen und sozialen Integration in die Aufnahmegesellschaft. Mit der Unternehmensgründung schaffen sich Selbständige ihren eigenen Arbeitsplatz und erfolgreich selbständig Erwerbstätige bieten mitunter Arbeitsplätze für andere. Gerade auch Klein- und Kleinstunternehmer/innen tragen dazu bei, kollektive Ressourcen in kooperativ-kreativer Weise zu mobilisieren. Auf diese Weise leisten sie wichtige Beiträge zur sozialen und ökonomischen Integration, zum gesellschaftlichen Aufstieg und zum Empowerment der involvierten (Familien-) Haushalte und der Diaspora-Communities generell.

Das Ausmaß selbständiger Erwerbstätigkeit in den Diaspora-Communities variiert erheblich. Angehörige bestimmter Herkunftsgruppen sind unternehmerisch aktiver als andere. Politische, strukturelle und ökonomische Rahmenbedingungen in den Städten, individuelle Ausbildungs- und Qualifikationsstrukturen sowie „hard“ und „soft skills” der Immigrant/inn/en spielen dabei als wesentliche Einflussfaktoren eine Rolle.

Aktuelle Daten belegen, dass mehr und mehr Personen mit Migrationshintergrund in Österreich unternehmerisch tätig werden (SchMATZ \& WeTZEL 2014). Von den 3,316.000 erwerbstätigen Personen ohne Migrationshintergrund 2019 waren 9,4\% Selbständige im nichtlandwirtschaftlichen Bereich. Bei den 1.003.100 erwerbstätigen Personen mit Migrationshintergrund lag der Anteil bei 8,0\% (STATISTIK Austria 2019, p. 61). ${ }^{1}$ Eingebürgerte werden in höherem Maße selbständig als ausländische Staatsbürger/ innen. Dies ist auch eine Auswirkung der legistischen Rahmenbedingungen, die es Drittstaatsangehörigen nur unter gewissen Auflagen erlauben, in Österreich Unternehmer/ innen zu werden. Der steigende Trend zur selbständigen Erwerbstätigkeit setzte in den 1980er Jahren ein und gewann seit den 2000er Jahren immer mehr an Dynamik. Damit wurde eine Entwicklung nachvollzogen, die in anderen Einwanderungsländern Europas schon mindestens eine Dekade zuvor begonnen hatte. Dabei zeigte sich aber auch, dass Immigrant/inn/en mit divergierenden Fähigkeiten und kulturellen Prägungen in unterschiedlichem Maße zu unternehmerischen Aktivitäten tendieren („Pull-Gründungen“)

1 Statistik Austria, Mikrozensus-Arbeitskräfteerhebung, Jahresdurchschnitt über alle Wochen. - Bevölkerung in Privathaushalten. 
und dass es sich bei den Unternehmer/inne/n mit Migrationshintergrund um eine äußerst heterogene Gruppe handelt (Aigner 2012; Alteneder \& WAgner-Pinter 2013).

Die Entscheidung für die Selbständigkeit erfolgt nicht immer freiwillig (,PushGründungen"), sondern kann auch eine Folge wirtschaftlicher Krisen, hoher Arbeitslosenquoten oder - gerade im Falle von Migrant/inn/en - von Diskriminierungserfahrungen in unselbständiger Beschäftigung sein. In Branchen mit hoher Beschäftigungslosigkeit ist sowohl bei Inländer/inne/n als auch Personen mit Migrationshintergrund die Selbständigenquote hoch. In Österreich sind Migrant/inn/en überdurchschnittlich oft im Handel und Reparaturgewerbe, in der Sparte Verkehr und Nachrichtenübermittlung, in den unternehmensorientierten Diensten, im Beherbergungs- und Gaststättenwesen sowie in den persönlichen Diensten (z.B. Betreuung und Pflege) selbständig (KOHLBACHER \& REEGER 2013).

Aufgrund divergierender Rahmenbedingungen ist die Migrant Entrepreneurship teilweise (aber nicht immer) durch andere Strukturen charakterisiert als das Unternehmertum von Nichtmigrant/inn/en. Dies betrifft die Gründungsneigung, die Altersstruktur der Unternehmer/innen, die Größe des Unternehmens, die Branchenzugehörigkeit, die Beschaffung des Anfangskapitals etc. Selbständige mit Migrationshintergrund verfügen über spezifische Ressourcen wie die Einbettung in transkulturelle Strukturen, familiäre Netzwerke und Netzwerke innerhalb der eigenen ethnischen Communities. Gleichzeitig werden Gründer/innen und Gewerbetreibende mit Migrationshintergrund mit vielen Hemmnissen konfrontiert: rechtliche Einschränkungen bei der Gewerbeausübung, Probleme bei der Fremdfinanzierung, sprachliche Barrieren beim Zugang zu Beratung und Förderprogrammen, Wettbewerbsnachteile etc. Häufiger sind unter Selbständigen mit Migrationshintergrund geringe Einkommenssicherung durch die selbständige Erwerbstätigkeit und höhere Insolvenzraten zu finden.

Die Expansion des Dienstleistungssektors hat eine Zunahme der Zahl der KMU (Kleine und mittlere Unternehmen) nach sich gezogen. KMUs nehmen in der urbanen Wirtschaftsentwicklung einen immer wichtigeren Stellenwert ein. Auf ihre Wirtschaftsleistung sowie Arbeits- und Ausbildungsplätze kann nicht verzichtet werden. Die von Selbständigen mit Migrationshintergrund geführten Unternehmen sind mehrheitlich der Kategorie der KMU zuzurechnen. Es befinden sich selbstverständlich aber auch größere Firmen und Ketten („DO \& CO“ oder „Türkis“ als Beispiele in der Gastronomie) darunter. Ein auch in international vergleichenden Analysen beobachtbares Charakteristikum der Migrant Entrepreneurship ist deren kleinbetriebliche Strukturierung mit geringer Kapitalausstattung, kleinen Anlagevermögen und wenigen Mitarbeiter/inne/n (European Commission 2002, 2007; Leicht et al. 2005, 2012). Darüber hinaus haben sich vielfältige Formen des Outsourcings und Subcontractings entwickelt. Unternehmer/ innen mit Migrationshintergrund gründen ihre Firmen häufiger als Vollerwerbsbetriebe und weisen oft eine höhere Risikobereitschaft auf als Inländer/innen. Dies führt jedoch auch zu hohen Fluktuationsraten zwischen den Branchen sowie häufigeren Betriebsschließungen. Angesichts der Prekarität vieler Unternehmen wird in der Forschung auch die Frage gestellt, inwieweit diese Dimension der unternehmerischen Selbständigkeit 
zur ökonomischen Integration beitragen kann. Im Hinblick auf die Integrationseffekte ist es primär wichtig, die Unternehmer/innen mit Migrationshintergrund als aktive Akteur/inn/e/n in den Fokus zu stellen.

Es ist eine in der Fachliteratur viel diskutierte Frage, inwieweit Unterschiede im Zugang zu und in der Ausprägung von Selbständigkeit nicht nur von sozialen, bildungsbezogenen und demographischen Faktoren, sondern auch von ethnischer Zugehörigkeit, Migrations- bzw. Fluchterfahrungen geprägt werden. Die Migrant Economy weist in den meisten europäischen Metropolen nicht nur Konzentrationen auf bestimmte Marktsegmente, sondern auch räumliche Konzentrationen auf. Da die Zuwanderung meist vor allem auf die Agglomerationen konzentriert ist, ist auch die Migrant Entrepreneurship vor allem im urbanen Kontext zu finden. In Österreich ist die Konzentration auf Wien besonders ausgeprägt. Stark gestiegen sind in Wien die Zahl und Vielfalt an Maßnahmen zur Förderung von Unternehmensgründungen von Migrant/inn/en als Unternehmer/innen. Somit steht fest, dass die Wichtigkeit dieses Teils der urbanen Ökonomie beträchtlich gestiegen ist.

Die Migrantenökonomie, die noch in den frühen 2000er-Jahren vor allem als ein Teilaspekt der urbanen „Superdiversity“ (vgl. VeRTOVEC 2009) betrachtet wurde, hat seither eine ausgeprägte Dynamik entwickelt. Während die individuellen Problemlagen der Unternehmer/innen in vielen europäischen Metropolen einigermaßen dokumentiert sind (DABRINGER \& TRUPP 2012), existieren nur wenige fundierte Studien zur ökonomischen und arbeitsmarktrelevanten Bedeutung dieses Teils der Wirtschaft. Im Vordergrund der öffentlichen Debatten zur Migrant Entrepreneurship stehen in erster Linie die spezifischen Bedürfnisse und Probleme dieser Unternehmen. Damit wird häufig der Bedarf an spezifischer Unterstützung assoziiert. Die Sichtbarkeit und Zahl dieser Unternehmen ist in den meisten Metropolen Europas kontinuierlich angestiegen. Damit steht die wirtschaftliche Bedeutung dieser Unternehmen außer Frage.

Der Genderaspekt und welche sozialen und (lokal-)politischen sowie im Falle selbständiger Frauen auch Empowerment-Effekte damit einhergehen, dies fand bislang weniger Beachtung (Brieger \& Gielnik 2021; Bruni et al. 2004; Colombelli 2019; DanNeCKer \& CAKIR 2016; Elam 2008; Halkias \& CARACATSANis 2011; Hancock et al. 2014; Hanson \& BlaKe 2009; Klyver \& Grant. 2010; Kofman et al. 2015; Kreide 2003; Leicht et al. 2009; Morocvasic 1991; Malach et al. 2010; Pio 2007; VerduiJn \& ESSERS 2013). Noch weniger bekannt ist über muslimische Frauen in ihrer Rolle als Unternehmerinnen und deren spezifische Herausforderungen (EsSER \& BENSHOP 2009; GARCIA-Diaz \& CARTER 2009). Dabei ist diese Relevanz für die urbane Ökonomie sowie die betreffenden Communities offenkundig: "The positive effects of entrepreneurship have been emphasised. However, promoting entrepreneurship is not an easy course. The sheer complexity of entrepreneurial processes should prevent us from envisaging entrepreneurship as a simple, one-dimensional phenomenon. A host of studies has sufficiently demonstrated that entrepreneurial success - and consequently the effects of it - is contingent on a plethora of social, political and economic conditions" (RATH \& SWAGERMAN 2011, p. 6). 
Es ist davon auszugehen, dass sich aus den komplexen Interdependenzen zwischen personen-, community- und umfeldbezogenen Faktoren Strategien ableiten, die sich in der Bedeutung, im Marktverhalten, ökonomischen Erfolg und den außerbetrieblichen Leistungen von Migrant/inn/enunternehmen widerspiegeln. $\mathrm{Zu}$ den individuellen und sozialen Konsequenzen der Migrant Entrepreneurship sind nur spärliche Befunde vorhanden. Dabei ist der Beitrag der Migrant Economy aus integrationspolitischer Sicht eindeutig positiv, obwohl der Schritt in die Selbständigkeit sowohl zu sozialem Aufstieg als auch zu prekären finanziellen Situationen, zu Selbstausbeutung oder sogar in eine Mobilitätsfalle führen kann (BAuBöcK 2001; GoEbel \& Pries 2006).

\subsection{Terminologie, theoretische Grundlagen}

\subsubsection{Migrant Entrepreneurship}

In der Literatur findet sich eine Vielzahl von theoretischen und analytischen Herausforderungen bei der Untersuchung von Migrant/inn/en, die von mehrdeutigen Begriffen und Definitionen bis hin zu unterschiedlichen nationalen Systemen der Bewertung, die Berichterstattung etc. reichen. Diese Komplexität bringt auch Unternehmer/innen hervor, die in keine der gängigen Kategorien fallen, was den vorhandenen Mangel an geeigneten Definitionen und Analysekriterien verdeutlicht. Nach RATH \& SWAGERMAN (2011, p. 12) "an entrepreneur is a person in effective control of a commercial undertaking for more than one client over a significant period of time. " Laut LIGHT \& KARAGEORGIS $(1994,648)$ besteht die ethnische Ökonomie aus ,, the ethnic selfemployed and employers, their unpaid family workers, and their co-ethnic employees".

Die seitens der Europäischen Union verwendeten Kategorien „migrant entrepreneur“ bzw. „migrant entrepreneurship“ (European Commission, August 2016, p. 9 ff.) haben sich im statistischen und politischen Sprachgebrauch durchgesetzt. Ein Teil der darin eingeschlossenen Unternehmer/innen ist selbst nicht zugewandert. Sie definieren sich häufig selbst nicht als Migrant/inn/en oder als Menschen mit Migrationshintergrund, jedoch durchaus als Angehörige einer bestimmten ethnischen Minderheit oder Diaspora. Damit bezieht sich der Terminus auf die definitorische Abgrenzung, wie sie durch die „United Nation Economic Commission for Europe“ (UNECE) unter Nutzung der Kriterien Staatsbürgerschaft und Geburtsland vorgenommen wurde (vgl. European Commission 2008; Segert 2010, p. 12). Seitens der EU wurde auch von „ethnic minority entrepreneurs“ gesprochen. Diese sind „entrepreneurs or potential entrepreneurs who are immigrants in the countries concerned. Immigrants are defined as persons who have been born abroad, irrespective of their nationality and whether they are considered ethnic minorities or not in the countries involved. 'Immigrants' also includes the offspring of immigrants, the second generation or the native born children of the first generation. In practice, the policy measures and support schemes rarely distinguish between generations of immigrants" (EUROPEAN COMMISSION 2008, p. 5). 
Im integrationskritischen Diskurs wir betont, dass diese Unternehmer/innen lediglich in ihrem (aktuellen) Aufenthaltsland einer ethnischen Minderheit bzw. Diaspora und nicht der dort sesshaften Mehrheitsgesellschaft angehören. Ihre ökonomischen Aktivitäten beziehen oft Herkunftsländer ein, in denen ihre Gruppe die Mehrheit bildet. Auch Begriffe wie jener der migrantischen Ökonomie/Migrantenökonomie sind kritisierbar, denn was ist noch ,migrantisch“ an Jungunternehmer/inne/n, die die zweite oder dritte Zuwanderergeneration repräsentieren und über keine eigenen Migrationserfahrungen verfügen? (vgl. BAYCAN-LEVEnT et al. 2009; BAYCAN-LEVEnT \& NiJKAMP 2007).

Es besteht kein allgemeiner Konsens über die Stellung von Unternehmer/ inne/n aus Diaspora-Communities und ihre Positionierung im Kontext der International Entrepreneurship, da sich die Ansichten über deren partielle Einbeziehung unterscheiden: "Diasporans who establish new ventures in their countries of origin comprise a special case of international ethnic entrepreneurship" (RIDDLE \& BRINKERHOFF 2011, p. 670). Dem gegenüber steht partielle Exklusion; "we exclude studies on transnational, ethnic and immigrant entrepreneurship from this review. Dies folgt DroRI et al. (2009, p. 1003), who observe that 'while in principle, transnational entrepreneurship is fundamentally compatible with IEs entrepreneurial initiative the approach and domain differ markedly from IE'," (JonES et al. 2011, p. 644).

\subsubsection{Ethnic Entrepreneurship}

In vielen Studien wurde der Terminus der „ethnischen Ökonomie“ verwendet. Dieser wurde von HiLlmann (1998, p. 4) als ,ein bestimmter Typus eines räumlichen Clusters von ethnischen, d.h. einer nicht-einheimischen Bevölkerungsgruppe zugehörigen, Unternehmen “ verstanden. Die STIFTUNG ZENTRUM FÜR TÜRKEISTUDIEN (1999; vgl. auch Aver 2008) bezeichnet damit die „, Gesamtheit des ökonomischen Handelns von Menschen unabhängig ihrer Staatsangehörigkeit und Ihres Geburtsortes, soweit sie im Vergleich zu Unternehmern aus der Mehrheitsgesellschaft unterscheidbare ethnische und kulturelle Merkmale aufweisen “. Nach Bosswick (2010, p. 15 f.) sowie RATH (2009, p. 10) “' 'ethnic entrepreneurs', are entrepreneurial persons who were born abroad as well as the second and third generation of immigrants“. Das „Ethnische“ der Ethnic Entrepreneurship ist ein Konstrukt aus Verbindungen oder Verhaltensmustern zwischen Personen, die einen gemeinsamen nationalen Hintergrund oder gemeinsame Migrationserfahrung haben (WALDINGER et al. 1990b, p. 34). Es handelt sich um jenen Teil der Wirtschaft, der durch Immigrant/inn/encommunities geprägt wurde. „Ethnic business" impliziert nicht notwendigerweise einen Migrationsvorgang, sondern beschreibt die Ökonomie einer ethnischen Minderheit (inkl. der zweiten Generation), die unter Umständen schon sehr lange in einem bestimmten Land ansässig sein kann. Die Grenze ist schwer zu ziehen, da „ethnic business“ mit der Gesamtwirtschaft in der Regel eng verflochten ist.

Der Begriff der „ethnischen Ökonomie“ wurde vielfach kritisiert, da damit eine simplifizierende Ethnisierung dieser vielfältigen Gruppen von Unternehmer/inne/n 
einhergeht (RATH \& Swagerman 2011, p. 11). Die neuere Forschung hat daher die Betonung des Zusammenhangs zwischen Unternehmertum und der ethnischen Identität abgeschwächt und unter anderem durch die Hervorhebung der Bedeutung der nationalen Zuwanderungsregime, die die Arbeitsmarktintegration von Immigrant/inn/en determinieren, ersetzt (BASSERMANn 2018). Hillmann, die besonders auch die Rolle der Frauen in der Selbständigkeit analysierte, stellte fest, dass sich die gängige Definition von „ethnic business“ implizit nur auf die Gruppe der männlichen Unternehmer - vor allem der ersten Zuwanderergeneration - bezog (1998, p. 34 ff., vgl. auch APITZSCH 2002). In vielen Studien wurde Ethnizität einfach mit dem Herkunftsland gleichgesetzt. Diese Etikettierung führte zu Simplifizierungen, die reflektierte Analysen eher behinderten (vgl. AldRICH \& WALDINGER 2000, p. 271) und die im konkreten Fall der kurdischen Herkunftsgruppe, die auf vier Nationalstaaten verteilt lebt, gar nicht anwendbar ist. Auch spielen die individuelle Migrationsbiographie und kulturelle Aspekte eine wesentliche Rolle. Sichtbar wird dies in den variierenden Anteilen unternehmerisch tätiger Frauen und Firmengründerinnen in unterschiedlichen Diasporas. HiLlmanN \& SOMmer (2011, p. 30) gingen davon aus, ,, dass die Unterschiede nur zu einem geringen Anteil kulturell bedingt und überwiegend sozioökonomischer Natur sind". Ethnische Zuschreibungen von herkunftsbezogenen Gruppencharakteristika sind aus heutiger Perspektive zu simplifizierend, um differenzierte Analysen zu ermöglichen. Es finden vielfältige Wechselwirkungen zwischen den kulturellen, sozialen und bildungsmäßigen Ressourcen in den Communities und den Opportunitätsstrukturen der Selbständigkeit statt.

Trotz der Kritik am Konzept der „Ethnizität“ (z.B. Fox \& DeMElza 2013; SöKEFELD 2007) erweist es sich zur Strukturierung von Problemstellungen und Forschungsfragen durchaus hilfreich, vor allem um gruppenspezifische Unterschiede im Umgang mit Opportunitätsstrukturen herauszuarbeiten. Die Strukturen von Selbständigkeit divergieren nach Herkunftsgruppen, was sich in der Branchenverteilung niederschlägt. Hillmann \& Sommer (2011, p. 10 ff.) führten die Unterschiede vor allem auf strukturelle und legistische Rahmenbedingungen für EU-Bürger/innen, Drittstaatsangehörige, Zuwanderung als Asylwerber/innen bzw. Geflüchtete oder Familiennachzug, Schlüsselarbeitskräfte etc. zurück. Dies erklärt jedoch nicht, warum sich beispielsweise die Branchenprofile auch zwischen Drittstaatsangehörigen aus verschiedenen Herkunftsländern unterscheiden. ${ }^{2}$

SCHULERI-HARTJE et al. sprachen von „selbständige(r) Erwerbstätigkeit von Personen mit Migrationshintergrund [...] und abhängige Beschäftigung in von Personen mit Migrationshintergrund geführten Betrieben, die in einem spezifischen Migrantenmilieu verwurzelt sind“ (2005, p. 21). „Personen mit Migrationshintergrund“ ist ein Begriff, der sich zunehmend in der medialen Diskussion und Berichterstattung durchsetzte, jedoch rezent inzwischen ebenfalls stark in der Kritik steht. Wie HerzoGPunZENBERGER et al. (2004, p. 9) anmerkten, ,verwandelt sich die Einfachheit dieser

2 Man vergleiche etwa die Branchenstruktur von Selbständigen aus Drittstaaten wie Indien oder China mit jenen aus der Türkei. 
... Einteilung ... in eine nicht länger brauchbare Kategorisierung, will man Prozesse verstehen, die generationenübergreifend mit dem Phänomen der Migration ... zu tun haben."

Migrant Entrepreneurs sind mit anderen Ausgangs- und Rahmenbedingungen konfrontiert als Selbständige ohne Migrationshintergrund. Diese Divergenz bewirkt zum Teil kulturell orientierte Interpretationen, die die unternehmerischen Aktivitäten von Migrant/inn/en vor allem auf die mit der Zuwanderung ,importierten“ Verhaltensmuster und Traditionen zurückführen. Dieser Zugang hat mittlerweile an wissenschaftlicher Erklärungskraft erheblich eingebüßt. Im Gegensatz dazu wird die Relevanz der Ressourcen ganzer Diaspora-Communities in den Vordergrund gestellt. Es sind nicht nur die personenbezogenen, sondern auch die sozialen und institutionellen Faktoren, welche Selbständigkeit fördern oder hemmen. Eine zentrale Rolle spielen die „modes of incorporation“" (PoRTES \& RUMBAUT 1990), d.h. die Bedingungen mit denen sich Immigrant/inn/en im Zielland auseinandersetzen müssen. Im Rahmen der Analysen von Migrant/inn/enselbständigkeit haben daher mehrdimensionale Konzepte an Bedeutung gewonnen (BIJEDić et al. 2017; Dana 2007; DANA et al. 2008; InSTITUT FÜR MitTELSTANDSFORSCHUNG, UniversitÄt MANNHEIM 2012; KaY \& Nielen 2020; Kloosterman \& Rath 2001, 2003; RaZin 2002; SACHs 2020; Westlund 2010; Zimmerman 2016). Resümierend ist zu sagen, dass eine exakte Quantifizierung der Wirkungsrelevanz des ethnischen Faktors gegenüber strukturellen, legistischen und sozioökonomischen Einflussgrößen nicht möglich ist. Die Zugehörigkeit zu einer Diaspora-Community kann, muss aber nicht eine gewisse Rolle im Kontext unternehmerischer Aktivitäten spielen (vgl. BEDNAR 2012).

\subsubsection{International Entrepreneur}

Ein weiterer Terminus ist jener des „International Entrepreneurs“6, dessen Konzeptualisierung eine grundlegende Frage für die Theorieentwicklung darstellt und kontroversielle Debatten bedingte, da die bereits früh in der EntrepreneurshipForschung von GARTNER (1989) gestellt und umstrittene Wer-Frage hinsichtlich Definitionen, Analyse und theoretischer Grundlagen herausfordernd bleibt (TABARES 2020). Die Mobilitätswende, die ansteigende internationale Migration und die Globalisierung (CAstles 2010; Faist 2013; Vertovec 2004) haben die Konzepte der Grenzziehung und ihre Bedeutungen sowie die unscharfe Unterscheidung zwischen nationalen und internationalen Kontexten in Frage gestellt. Ältere, starre Rahmenwerke für die Annäherung an das Phänomen des International Entrepreneurs wurden damit ungeeignet. Die Prozesse der unternehmerischen Internationalisierung haben sich weiterentwickelt, um neue Aspekte zu berücksichtigen. Diese realen Aspekte erfordern eine neuartige und vertiefte Betrachtung der jeweiligen Kontexte unternehmerischen Handelns und der Unternehmer/innen als Akteur/inn/e/n, z. B. hinsichtlich der jeweiligen Voraussetzungen, Abläufe und Verhaltensweisen (vgl. DroRI et al. 2009; Elo 2016). Unternehmerische Verhaltensschemata entwickeln sich weiter und internationale Chancen werden heute 
anders adressiert, generiert und wahrgenommen als noch vor einigen Jahren, wobei auf aggregierten Ebenen institutionelle und systemische Rahmenbedingungen eine wichtige Rolle spielen (BRINKERHOFF 2016).

\subsubsection{Refugee Entrepreneurship}

Die Entrepreneurship von Geflüchteten (Refugee Entrepreneurship) weist zwar einige Spezifika auf, z.B. die in der Regel nicht vorhandene Kapitalbasis, der rechtliche Status etc., teilt jedoch viele Charakteristika mit den Konzepten der Migrant und der Diaspora Entrepreneurship (vgl. BizRi 2016; Quoc 2020; Desai et al. 2021, HeILBRUNn et al. 2019). Der Bezug zur Refugee Entrepreneurship für vorliegende Untersuchung resultiert aus dem Faktum, dass sich unter den kurdischen Unternehmer/ inne/n etliche befinden, die aufgrund politischer Verfolgung (Kurd/inn/en aus der Türkei bereit seit den 1970er-Jahren) und auf dem Wege der Flucht (Kurd/inn/en aus Syrien v.a. 2015) nach Österreich gelangten.

\subsubsection{Diaspora und Diaspora Entrepreneurship}

\subsubsection{Diaspora}

Der Begriff „Diaspora“ hielt in den späten 1980er Jahren Einzug in den kulturwissenschaftlichen Diskurs, traditionell war er ausschließlich auf die zerstreuten jüdischen Gemeinschaften fokussiert. Danach erhielt der Terminus einen zunehmend umfassenderen und komplexeren Bedeutungsgehalt (Кокот 2002, p. 30), indem eine größere Zahl von durch Immigration entstandener Communities mit diesem Terminus beschrieben wird. Einige Communities verwenden ihn zudem auch als Eigenbeschreibung, um sich von den kategorisierenden und inferiorisierenden Fremdbeschreibungen als „Ausländer/ innen“, Immigrant/inn/en oder Gruppen mit Migrationshintergrund durch Teile der Aufnahmegesellschaften zu distanzieren. Der weit gefasste Gebrauch des Begriffs bedingte in der Forschung immer mehr Debatten über dessen wissenschaftlichen Nutzen und seiner analytischen Unschärfe. So wurden innovativere Konzepte zum modifizierten Gebrauch oder basierend auf selektiven Definitionsmerkmalen vorgeschlagen, um damit analytische Fortschritte zu erzielen (BRAKEMEIER-BORREK 2005; STADLER 2009).

MARIENSTRAS bezeichnete als Diaspora ,, any community that has emigrated whose numbers make it visible in the host community " (ibid. 1989, p. 125). Eine einheitliche und konsistente Theorie der Diaspora existiert bis heute nicht „höchstens Theoriebruchstücke, eben Ansätze“" (Hettlage 1993, p. 76). Diese wurden der Migrations-, Ethnizitäts- und Identitätsforschung entnommen. Der Diasporabegriff wird oft in fast synonymer Verbindung mit den Konzepten der ethnischen Minderheit, der Ethnizität sowie in Anlehnung an Transnationalität oder Nationalismus verwendet (ALINIA 2004, p. 27).

Vertretern einer kritischen Perspektive erschien die „Diaspora“ - eine Gruppe und Kultur, die nicht fixiert und unbeweglich ist - als Fortschritt gegenüber Vorstel- 
lungen von Ethnizität und ethnischen Gruppen. Vor allem in den britischen Cultural Studies war das Konzept beliebt, da "Diaspora is a valuable idea because [it is] [...] an alternative to the metaphysics of 'race', nation and bounded culture" (GILROY 1997, p. 328) und "[it] puts emphasis on contingency, indeterminancy and conflict" (ibid, p. 334). Ähnlich argumentierte auch HaLl: "The diaspora experience ... is defined not by essence or purity, but by the recognition of a necessary heterogeneity and diversity; by a conception of 'identity' which jives with and through, not despite, difference; by hybridity. Diaspora identities are those which are constantly producing and reproducing themselves anew, through transformation and difference." (ibid. 1990, p. 235).

SAFRAN kritisierte, dass „Diaspora“ eine nur metaphorische Kategorie ohne präzisen analytischen Wert darstellte (1991, p. 83). Die Kritik an der Ausweitung des Konzeptes wird von vielen Autor/inn/en geteilt, die auch Modifizierungen und exaktere Definitionen vorschlagen. Wichtig für die jüngere Diskussion war SAFRANs Liste zentraler Merkmale, die Diaspora von Konzepten wie Minderheit oder Ethnizität/ethnische Gruppe abgrenzten (SAFRAN 1991, p. 83f.) James CLIFFORD (1994) verfolgte die umgekehrte Definitionsstrategie, indem er bestimmte, was Diaspora nicht ist und dies abgrenzte, nämlich der Nationalstaat mit seiner Territorialität, indigene bzw. autochthone Gruppen, die ebenfalls lokalisiert sind, sowie Immigrant/inn/en, von denen im Gegensatz zur Diaspora Assimilation erwartet wird. Die Abgrenzung zwischen Diaspora und Nationalstaat war demgemäß besonders wichtig. Khachig TÖLÖLYAN (1991) bezeichnete Diaspora als das transnationale „Andere“ des Nationalstaats. TÖLÖLYAN (1996) bemühte sich um eine Begriffsbestimmung und unterstrich, dass Diaspora auf individuellen und kollektiven Akten der Unterstützung und Aufrechterhaltung von gemeinschaftlichen Institutionen der eigenen Gruppe basiert, womit Assimilation in die Aufnahmegesellschaft verhindert wird (1996, p. 15).

Signifikante Merkmale einer „modernen“ Diaspora nannte MoosMüLLER (2002a, b), unter Bezugnahme auf Clifford (1994), Cohen (1997, 2008), Hettlage (1993). CoHens Typologie widersprach Кокот (2002), welche intensive Feldforschungen zum Begriff der Diaspora durchführte und von offenen Arbeitsdefinitionen zum Begriff Diaspora im Dreiecksverhältnis Diaspora, Residenzland und Heimatland ausging: „,... alle Gruppen, die von einem Zentrum aus in mehr als einem Ort verstreut sind und die ein Bewusstsein kultureller Gemeinsamkeit und soziale Vernetzungen mit anderen Gemeinden der selben Diaspora aktiv aufrechterhalten " (Кокот 2002, p. 35). Relevante Elemente sind das Element der Zerstreuung, die Selbstwahrnehmung als kulturelle Gemeinschaft und die Praxis sozialer Beziehungen (institutionelle Ebene und/oder Ebene persönlicher Netzwerkbeziehungen). Sie definiert Diaspora aufgrund ihrer Ergebnisse als ,,Paradigma für alle Formen von Migration [...] über Generationen hinweg - transnationale und weltweite Netzwerke, die eigenständige kulturelle Identitäten aufrechterhalten. " $\mathrm{Zu}$ den entscheidenden Faktoren gehören auch der Bezug auf eine gemeinsame Geschichte und sehr oft auf die Religion sowie die Lokalität, nämlich Örtlichkeit als einschränkende Bedingung sowie symbolische Ortsbezogenheit (Кокот 2002). 
BRUBAKER (2005) sprach sogar von der „Diaspora Diaspora“ und kritisierte die semantische, konzeptuelle und disziplinäre Zersplitterung der Bedeutungen von Diaspora (2005, p. 1). Der Begriff werde für alle möglichen Gemeinschaften benutzt. Einzige verbindende Kriterien sind die räumliche Zerstreuung und das Gefühl einer aus der Heimat mitgebrachten Gemeinsamkeit (2005, p. 3). Im Zuge der Einpassung des Begriffs in die diversesten politischen oder kulturellen Kontexte verliert er sein Potenzial zur Deskription konkreter Gruppen und Phänomene: ,If everyone is diasporic, then no one is distinctively so “ (ibid.). BRUBAKER schlug als Alternative vor, Diaspora nicht als Terminus zur Etikettierung einer „Gruppe“ oder „Gemeinschaft“ zu benutzen, sondern als ein Konzept politischer Praxis, mit dem politische Ansprüche ausgedrückt werden.

Migrant/inn/en halten multiple Zugehörigkeiten zu Räumen und Gesellschaften aufrecht (vgl. VAN HEAR 1998, p. 4). Migrant/inn/engruppen sind auch politische Akteur/inn/e/n und stellen, zum Teil jedenfalls, zuvor selbstverständliche Forderungen nach Assimilation in Frage. Dies verändert auch den politischen Diskurs in den Aufnahmegesellschaften. Gruppengrenzen werden gleichzeitig diskursiv betont, wie in Frage gestellt. Die Homogenität einer Gesellschaft existiert nicht. In sozialwissenschaftlichen Diskursen steht der Diasporabegriff in einem Spannungsfeld zwischen Ausweitung und Ansätzen zur Konkretisierung. Die nach BRUBAKER politische Verwendung des Konzeptes muss aber aus sozialwissenschaftlicher Perspektive reflektiert werden.

Anthias (1998, p. 557 ff.) sah Diaspora als essentialistisches Konzept an, weil der Begriff Menschen dauerhaft einer Herkunft zuschreibt und damit kontinuierlich als ,anders/fremd“ markiert. Damit wird die ethnische Differenz betont, während andere Differenzen wie Gender oder Klassenzugehörigkeit keine Beachtung finden. Kultur wird dann zwar nicht mehr mit einem Territorium verbunden, aber mit einer bestimmten Herkunft fixiert. Damit kritisiert AnTHIAS, dass das Konzept von Diasporagemeinschaften ähnlich homogenisierend funktioniert, wie Konzepte von Ethnizität und ethnischen Gruppen (HeINZ 1993). Gemeinsame Herkunft ist nur eine Selbstund/oder Fremdzuschreibung und wird massiv essentialisiert. Hinzu kommt, dass die Zuschreibung von Herkunft alles andere als eindeutig ist.

Soysal hob hervor, dass der Diasporabegriff die Vorstellung fester Zugehörigkeit zu einem bestimmten Ort festschreibt, der aber verlassen wurde: „Diaspora is the extension of the place left behind, the ,home: Thus, the presumed rootlessness of immigrant populations in the here and now of the diaspora, and their perpetual longing for then and there. Theoretical move, that is, designating immnigrant populations as diasporas, ignores the historical contingency of the nation-state, identity and community and reifies them as natural" (Soysal 2000, p. 3).

VERTOVEC (1997, p. 1) kritisierte einerseits den „over-use“ und die gleichzeitige „under-theorisation“ des Konzepts, welches dazu dienen sollte, bestimmte Sachverhalte analytisch hervorzuheben und deskriptiv zu beschreiben, anstatt sie in eine Menge sehr ähnlicher Konzepte aufgehen zu lassen. Um einen Überblick über die gängigen wissenschaftlichen Verwendungsweisen zu geben, identifiziert er die drei geläufigsten und 
in der Literatur am häufigsten vorkommenden Bedeutungen von Diaspora, die dessen konzeptuellen Nutzen widerspiegeln (ibid., p. 2). Er versuchte nicht, Diaspora an sich $\mathrm{zu}$ definieren, sondern identifizierte drei vorherrschende Bedeutungen des Diasporakonzeptes in der Literatur. Dies sind Diaspora als (1) soziales Konstrukt, als (2) spezifische Qualität von Bewusstsein und als (3) Modus kultureller Produktion.

Diaspora als ,social form“ orientiert sich an den jüdischen Gemeinschaften als Prototyp. Es besteht eine Beziehung zwischen a) verstreuten ethnischen Gemeinschaften, die sich selbst als solche identifizieren, b) den territorialen Kontexten und Aufnahmegesellschaften, in denen sie sich aufhalten, c) der Zuschreibung eines inferioren Status und d) den Herkunftsländern aus denen Einwanderer stammen (ibid., p. 5). Die Diaspora als soziale Form ist durch die transnationalen sozialen Netzwerke zwischen den zerstreuten Gruppen, durch das Anderssein in der Aufnahmegesellschaft, durch eine Sehnsucht nach der Heimat und etwaige Rückkehrwünsche gekennzeichnet. Das Diasporakonzept bezieht sich auch auf die Selbstwahrnehmung (,type of consciousness“) von Angehörigen bestimmter Gruppen innerhalb einer transnationalen Community. Einerseits sind sich Individuen in Diasporas ihrer Multilokalität bewusst, andererseits fühlen sich manche hin- und hergerissen zwischen der Aufnahmegesellschaft, in die sie sich integrieren möchten, und dem Herkunftsland. Das spezifische Bewusstsein entsteht durch einen dualen Prozess: Zuerst wird die eigene Identität durch Erfahrungen wie Xenophobie, Diskriminierung und Ausgeschlossenheit durch die umgebende Gesellschaft (ibid., p. 8) in Frage gestellt. Im Rahmen einer Selbstverortung kommt es zu einer stärkeren Zuwendung hin zu den eigenen kulturellen und ethnischen Wurzeln. Damit kann eine positive Identität konstruiert werden (Alinia 2004, 250; Alinia et al. 2014). Nicht selten kommt es in diesem Prozess zu einer idealisierten und stereotypisierten Vorstellung von der ,eigenen Kultur“ und einer ethnisch geprägten Gemeinschaft (VerTOVEC 1997, p. 12). Diaspora als Modus kultureller Produktion fokussiert nicht auf Gemeinschaften, sondern auf Räume, Stätten und Akteur/inn/e/n kultureller Kreativität, die kulturelle Inspirationen nutzen.

Wenn Diaspora nicht automatisch auf „Herkunft“ und Migration basiert, stellt sich die Frage, wie sie denn entsteht. Diaspora entsteht nicht von selbst, sondern Akteur/inn/e/n müssen eine Diaspora also auf Basis von Prozessen der sozialen und politischen Mobilisierung ,erzeugen“, die ihrerseits eine ,vorgestellte transnationale Gemeinschaft" (SöKEFELD 2011, p. 28; 2008) hervorbringen. Das bedeutet, dass sich eine Gemeinschaft als ,eigene“ Gemeinschaft vorstellt, als getrennt und unterschieden von anderen (ibid.). Eine gewisse Differenz ist somit impliziert, womit auch das Konzept der „Identität“" ins Spiel kommt.

Seit TÖNNIES‘ Gegenüberstellung von Gemeinschaft und Gesellschaft (TöNNIES 2005) wurde „Gemeinschaft“ vor allem als soziologischer Gruppentypus betrachtet. Gemeinschaft als soziale Struktur und Gemeinschaft als symbolische Konstruktion sind nicht zwingend ident. Es müsste jeweils empirisch belegt werden, ob eine symbolische, kulturelle Konstruktion, also eine „Vorstellung“ von Gemeinschaft, tatsächlich 
auf sozialen Gemeinschaftsbeziehungen basiert, denn Nichtkongruenz ist möglich. Die Frage des Zusammenhangs von Sozialbeziehungen und symbolischer Gemeinschaft wird dann zu einer Frage der sozialen Mobilisierung für bestimmte symbolische Konzepte von Gemeinschaft, die empirisch mit sozialwissenschaftlichen Methoden analysiert werden können (SöKEFELD 2011, p. 29). Folgende Fragen müssen an die Konstruktion von Diaspora als vorgestellter transnationaler Gemeinschaft gestellt werden: Wer propagiert welche symbolische Konstruktion von Gemeinschaft? Wer lässt sich für welche symbolische Konstruktion von Gemeinschaft mobilisieren? Welche Konflikte bestehen zwischen unterschiedlichen Konstruktionen von Gemeinschaft und in welchem Zusammenhang stehen sie mit anderen Auseinandersetzungen, etwa um Macht und Anhängerschaft? In welchem gesellschaftlichen und politischen Rahmen finden diese Auseinandersetzungen statt? Damit kann die Prozesshaftigkeit von Diaspora erfasst werden, und eine Essentialisierung des Konzeptes wird verhindert, die sich darin manifestiert, eine Diaspora fest an ein „Herkunftsland“ zu assoziieren (SöKEFELD 2011, p. 30).

Diaspora ist daher keine automatische Folge von Migration, sondern entsteht durch Prozesse der sozialen, politischen und kulturellen Mobilisierung, die bestimmte Vorstellungen von Gemeinschaft propagieren und sozial wirksam werden lassen. Dies geschieht oft, indem „Peers“ Ideen von Gemeinschaft entwickeln und verbreiten, Anhänger für diese Vorstellung gewinnen und sie mit politischen Parteien oder Vereinen untermauern. Damit werden Menschen für eine Diaspora mobilisiert. Diese Prozesse lassen sich mithilfe von Konzepten der Theorie sozialer Bewegungen untersuchen (vgl. SöKEFELD 2006, p. 30 f.). In der Geschichte von Diasporagemeinschaften treten häufig wellenförmige Bewegungen auf, in denen abhängig von herausfordernden Ereignissen und Entwicklungen Phasen starker oder schwächerer Mobilisierung einander abwechseln. Der Fokus auf der Mobilisierungsperspektive erlaubt auch eine Differenzierung zwischen Angehörigen einer Diaspora. Nicht alle sind für eine Diaspora gleichermaßen engagiert, es gibt Aktivist/inn/en und jene, die sich bestenfalls mobilisieren lassen. Nicht jedes Individuum in einer Diaspora Community ist gleichermaßen „diasporisch“. Damit gestattet dieses Konzept von Diaspora als Prozess sozialer Mobilisierung konkurrierende Modelle von Gemeinschaft und unterschiedliche Bewegungen zur Mobilisierung zu kombinieren (SöKEFELD 2006, 2008).

Ein Teil der Community Studies arbeitet mit vordefinierten Definitionen des Begriffs „Community“. Ohne hier eine ausführliche Diskussion über die für Migrantengruppen relevanten sozialen und kulturellen Grenzziehungen führen zu können, kann festgehalten werden, dass der integrierte Intersektionalitätsansatz bewusst für nicht-finale Rekonstruktionen der beteiligten sozialen Strukturen und Prozesse plädiert. Dies ist umso notwendiger, als der Community-Ansatz die Migranten-Community als eine quasi „natürliche“ Kategorie behandelt, die sich aus der Tatsache ergibt, dass die Diaspora und ihre transnationalen sozialen Räume fast zwangsläufig mit der Bildung sozialer Enklaven innerhalb „fremder“ Gesellschaften, d.h. der autochthonen Gesellschaften an den Zielorten, zusammenfallen. Daher erscheinen nationale, konfessionelle oder ethnische Gemeinschaften oder sogar als homogen gedachte „Parallelgesell- 
schaften“ (JACKSON \& PARKes 2007; HESs 2008) als fast unvermeidliche Repräsentationen von Diaspora-Gruppen, während wahrscheinlich viel komplexere Mechanismen der Konstituierung von Communities relevant sind.

Eine Lösung, die der Intersektionalitätsansatz für das Insider-Outsider-Problem bei den Diasporas anbietet, bezieht sich nicht nur auf das Konstrukt der ethnischen Gemeinschaft, sondern gilt auch für die Definition von Gemeinschaften und Untergruppen nach jedem anderen Kriterium: So beziehen sich viele Studien zu den urbanen Modi der sozialen Inklusion bzw. Exklusion auf sogenannte ,problematische“"Gruppen, die eine Häufung verschiedener Merkmale der Unterprivilegierung oder Marginalisierung aufweisen. Neben einer inhärent strukturellen Sicht von außen, die diese Gruppen als strukturell benachteiligt im Vergleich zu den anderen Gruppen der Gesellschaft beschreibt, ist der Intersektionalitätsansatz in der Lage, eine spezifische Optik zu entwickeln, die sich auch auf die interne Struktur der Gruppe konzentriert. Er ermöglicht deshalb den analytischen Blick für kulturelle Hybridisierung und fließende Selbstdefinitionen von Individuen und vermeidet damit vorgefasste, willkürliche Vorstellungen von In- bzw. Outsidern. Dies impliziert auch theoretische Verknüpfungen zu den Konzepten der Identity as Transition (ForTIER 2000, p. 8; 2006, p. 65) oder als Rahmen für den Übergang von einem sozialen Raum in einen anderen, der den Wunsch nach Zugehörigkeit und nicht nach einer bereits konstituierten und oft essentialisierten Identität ausdrückt (РROBYN 1996, p. 23). Der Ansatz der Intersektionalität berücksichtigt, dass auch Individuen, die in stark segregierten Gemeinschaften leben, dazu neigen, Vergleichs- oder Referenzrahmen aufzubauen, die sich an der Welt der Mehrheitsgesellschaft außerhalb der Diaspora orientieren. Dennoch kann das alltägliche Leben sehr stark auf Netzwerke und Milieus beschränkt sein, die als ,ethnisch“ klassifiziert werden können.

\subsubsection{Diaspora Entrepreneurship}

In der Fachliteratur tritt der Terminus der „diaspora entrepreneurship“ als Subkategorie der "transnational entrepreneurship“ auf, wobei das Konzept der Transnationalität auf VerTOVEC (2009) zurückgeht. Tatsächlich war der moderne Transnationalismus und waren transnationale Lebensformen dafür verantwortlich, dass unterschiedliche Typen von Unternehmer/inne/n mit divergierenden Motivationen, Migrationserfahrungen etc. entstanden. RIDDLE et al. (2010, p. 398) definieren DiasporaUnternehmer/innen als "migrants and their descendants who establish entrepreneurial activities that span the national business environments of their countries of origin and countries of residence”. ,Transnational entrepreneurship“ bezieht sich auf Unternehmer/innen mit multiplen räumlichen, sozialen und kulturellen Verflechtungen, die Opportunitätsstrukturen nutzen, die aus wirtschaftlicher Globalisierung sowie grenzüberschreitenden unternehmerischen Aktivitäten resultieren (MORAwsKA 2004; DRORI et al. 2009; Honig \& Drori 2010).

Transnationalismus, Globalisierung, technologische Entwicklungen im Sektor der Transport- und Kommunikationstechnologien haben Migrationsbewegungen und 
Diasporaentwicklungen weltweit stark forciert (LEVITT 2001; TöLÖLYAN 1996). Die Diaspora-Entrepreneurship bezeichnet unternehmerische Aktivitäten, die von Angehörigen so genannter Diaspora-Communities geleistet werden. Diaspora-Communities sind Immigrant/inn/en sowie Angehörige der zweiten und weiterer Generationen, die nach SAFRAN (1991) starke Beziehungen zu ihrem Herkunftsland aufrechterhalten. Diese Unternehmer/innen stehen in besonderen kulturellen Konstellationen, da sie in mehrere kulturelle Kontexte, Gesellschaften und Staaten eingebettet sind (KLOOSTERMAN \& RATH 1999). Sie agieren in komplexen transnationalen kulturellen, institutionellen und wirtschaftlichen Konfigurationen, welche es den betreffenden Selbständigen erlauben, geschäftliche Chancen sowohl in den Herkunfts- als auch in den Migrationszielländern optimal zu nutzen (Drori et al. 2010; Kasemsap 2017). Darüber hinaus leistet die Diaspora Entrepreneurship einen wichtigen Beitrag zur Entwicklung der jeweiligen Diaspora Community, in welche sie eingebettet ist (BRINKERHOFF 2008). Zwar waren die mit der Kategorie der Diaspora einhergehenden Konnotationen ursprünglich eher negativ, da sie häufig mit Vertreibung, Vulnerabilität, Entfremdung und ökonomischer Marginalität assoziiert waren (HiLlmAnn 2011 a, b; Vertovec 2005), zunehmend hat sich die Wissenschaft aber stärker den positiven Aspekten des Diaspora-Phänomens zugewandt.

Es existiert bislang kein klares, kohärentes Bild der Diaspora-Entrepreneurs, was vor allem auf die Heterogenität des Diasporaphänomens zurückzuführen ist. Frühe Analysen haben sich meist auf jeweils einen bestimmten Typ der Diaspora konzentriert, nämlich jene aus so genannten Entwicklungs- oder Schwellenländern. Dieser Typ von Unternehmer/inne/n kann die sehr heterogene Gruppe jedoch kaum repräsentieren. Beispielsweise handelt es sich bei „Ascending Diaspora Entrepreneurship“ um Unternehmer/innen, die aus wirtschaftlich weniger entwickelten Kontexten in höher entwickelte, wirtschaftlich stärkere ziehen. Den Gegensatz bilden die „Descending Diaspora Entrepreneurship (Harima 2014, p. 65 ff.). Der Terminus „Diaspora“ wurde in der Literatur auch synonym für die betreffenden Bevölkerungsgruppen verwendet. Im Zusammenhang damit steht, dass einige Forscher/innen Diaspora-Entrepreneurs fast in einer Opferrolle gefangen sahen, also getrieben von ökonomischen Notwendigkeiten aufgrund eines Mangels an Arbeitsplätzen, wenig Alternativen und kaum vorhandenen finanziellen Ressourcen. Zwar können solche Situationen in der wirtschaftlichen Realität tatsächlich auftreten und sie können auch den Gang in die Selbständigkeit wesentlich verursacht haben, diese prekären Rahmenbedingungen und Unternehmer/ innen sind jedoch nur eine Facette eines weitaus komplexeren Phänomens.

In den Diaspora-Communities besteht häufig eine höhere Gründungsneigung als in der Bevölkerung der Migrationszielländer (CFE \& DueDıL 2014). Analysen haben viele positive Aspekte der Diaspora-Entrepreneurship nachgewiesen, wie z. B. den Transfer von Informationen und Technologien (SAXENIAN 2002, 2005; Tung 2008), die Förderung der wirtschaftlichen Entwicklung als Folge von Investitionen im Herkunftsland (BARNARD \& PENDOCK 2013) sowie die von den Diaspora-Communities ausgehenden Stimuli für die lokale Wirtschaft (PorTes et al. 2002). Aufgrund erheb- 
licher sozialer und wirtschaftlicher Relevanz im Rahmen transnationaler Strukturen ist die Diaspora-Entrepreneurship auch für politische Entscheidungsträger/innen von Interesse. Es ist anzunehmen, dass die Bedeutung dieses Segments der Wirtschaft in Zukunft noch zunehmen wird. Trotz deren Wichtigkeit (DuTIA 2012; ELO 2014) wurde die Vielfalt der Diaspora-Entrepreneurship noch wenig untersucht. Diaspora-Communities sind stets sehr komplex, da sie die unterschiedlichsten Personen umfassen (vgl. CoHEN 1997, 2008). Zum Beispiel können sowohl Kriegsflüchtlinge als auch Eliten angehörende Expatriates in eine Diaspora eingeordnet werden. Diese unterscheiden sich allerdings in zahlreichen Aspekten voneinander, wie unternehmerischen Motivationsstrukturen (Notwendigkeit versus Gelegenheitsunternehmertum), verfügbaren Ressourcen, sozialem, kulturellem und ökonomischem Kapital sowie den institutionellen Rahmenbedingungen.

Trotz des steigenden Forschungsinteresses fehlen immer noch Daten und Analysen über zahlreiche Aspekte der unternehmerischen Aktivitäten von DiasporaEntrepreneurs. Die fundamentale Bedeutung sozialer Netzwerke zählt zu den am besten untersuchten Phänomenen. Aufgrund ihrer „mixed embeddedness“ (KLoosterman et al. 1999; JONES et al. 2014) verfügen Diasporas über diversifizierte Netzwerke in den Herkunfts- und Aufenthaltsländern (KuZnetsov 2006). Aus diesem Grund wurde die Netzwerkdynamik als ein wichtiger Faktor für ihre Geschäftsaktivitäten angesehen. Insbesondere ethnische Netzwerke außerhalb der Heimatländer werden als „DiasporaNetzwerke" bezeichnet und die jeweiligen Netzwerkdynamiken analysiert (KuZNETSOV 2006; KuZnetsov \& SABEL 2008). Einigkeit herrscht weitgehend darüber, dass für die Entrepreneurship sogenannte Diaspora-Netzwerke wichtige Auswirkungen auf die transnationalen ökonomischen Aktivitäten haben, z. B. hinsichtlich der Rekrutierung von Beschäftigten (Light 2000), der Kapitalbeschaffung (SAXENian 2002), der Informationsflüsse (MEYer \& WatTiaux 2006) und last, but not least der Role Models und ihrer Veränderungen (Toledano \& URBANO 2008).

Die Betonung liegt auf der vielfältigen und wichtigen Rolle der selbständig Erwerbstätigen für die Communities, in die sie eingebettet sind, hinsichtlich transnationaler Kapital- und Knowhowtransfers, Arbeitsplatzbeschaffung und der Etablierung von Wirtschaftsbeziehungen (PorTes et al. 2002; RidDLE et al. 2010). Die Netzwerke der Diaspora bieten grenzüberschreitende Kontakte, Informationsdiffusion und Marktchancen. Vorliegende Untersuchungen haben auch die ökonomischen und sozialen Entwicklungsimpulse in den Herkunftsstaaten sowie den Aspekt der politischen Mobilisierung und der Nutzung transnationaler wirtschaftlicher Ressourcen nachgewiesen (Kitching et al. 2009; Terjesen \& Elam, 2009; Sepulveda et al. 2010; Riddle \& BrinKerhofF 2011). „Remittances“ der Migrant/inn/en an Angehörige in Herkunftsländern, Investitionen und humanitäre Unterstützung sowie - im Falle der kurdischen Diaspora besonders wichtig - politische Unterstützung angesichts der vielfältigen Unterdrückungssituationen dieser Minderheit in den Herkunftsstaaten spielen eine wichtige Rolle (Ionescu 2006; KuZnetsov \& Sabel 2006; Brinkerhoff 2008; Newland \& TANaka 2011; SyretT \& SePulveda 2011). Diaspora Entrepreneurs tätigen aus unter- 
schiedlichen Motivationen und nicht nur aus finanziellen Gründen in ihren Herkunftsländern Investitionen (GillesPie et al. 1999; NiELSEN \& Riddle 2010; Riddle et al. 2010; BRINKERHOFF 2016). Transnationale formelle und informelle Vernetzungen sowie Opportunitätsstrukturen der Beziehungen zwischen Diaspora-Communities werden durch Faktoren beeinflusst, die sich von den Spezifika in den Herkunfts- und Aufnahmeländern ableiten (AHMAdov \& SASSE 2016; GuARnizo et al. 2003; KAPUR 2010). Vorliegende Studien (etwa Elo 2016; Riddle et al. 2010; Sharma \& Montes 2013) haben Unterschiede der Diaspora Entrepreneurship zu anderen Formen des transnationalen Unternehmer/innentums teilweise herausgearbeitet.

Diaspora-Entrepreneurs sichern sich durch ihre Tätigkeit eine ,upward social mobility" und bringen auch diverse Formen des Sozialkapitals in ihre Communities ein. Unternehmer/innen signalisieren sozialen Aufstieg und zeigen der Aufnahmegesellschaft und ihrer Community als Role Models ökonomischen Erfolg. Sie stehen sozusagen als „Brückenbauer“ zwischen der Diaspora, welcher sie angehören, und Kund/inn/en, Lieferant/inn/en etc. aus der Mehrheitsgesellschaft. Diese (Ver-) Mittlerposition prädestiniert sie auch, in Organisationen der Diaspora als Repräsentant/ inn/en zu fungieren.

Jedenfalls noch kaum untersucht wurde, inwieweit Branchenwahl, Unternehmensführung und geschäftliche Praxis in einer Relation stehen zur Zugehörigkeit zum sozialen Kollektiv der Diaspora, welches gerade im Falle der Kurd/ inn/en über mehrere Staatsgrenzen hinweg existiert und eine spezielle ethnische Identität in Verbindung mit explizit politischen Gruppeninteressen kombiniert. Dies gilt auch für die Beziehungen der unternehmerischen Aktivitäten zu den typischen Besonderheiten der betreffenden Diaspora und welche Rolle der Entrepreneurship außer- und innerhalb der jeweiligen Diaspora zukommt. Dazu bedürfte es systematischen Analysen aktueller Entwicklungen in der betreffenden Community im Zusammenhang mit der Konstruktion und Bewahrung der eigenen diasporischen Identität, transnationalen Verflechtungen und Organisationsformen (z.B. lokalen Vereinen) der Diaspora in ihren Interdependenzen zur Entrepreneurship.

\subsubsection{Die Diaspora Entrepreneurship - ein urbanes Phänomen}

In den USA gewannen bereits in den 1970er Jahre klein strukturierte Unternehmen wieder an Bedeutung. Diese Entwicklung setzte mit zeitlicher Verzögerung in Großbritannien und dann in den Niederlanden ein. Mit Beginn der 1980er Jahre verstärkte sich das Interesse an der unternehmerischen Selbständigkeit von Immigrant/inn/en und eine Forschungstätigkeit entwickelte sich. Diese war in ihrer Anfangsphase weniger von der Migrationsforschung getragen, sondern von einem wirtschaftssoziologischen Interesse im Rahmen der Kleinunternehmerforschung. Zunehmend entwickelte sich ein Forschungszugang, der sich stärker am Interesse der Funktionen „ethnischer Kolonien“, der Diasporas und der Migrant Economy orientierte (vgl. IKONOMU 1989).

Großbritannien war das erste europäische Land, das die „ethnic minorities“ und deren Ökonomien als wesentlichen Part urbaner Ökonomien „,entdeckte“. Der Hintergrund 
dafür war ein pragmatischer: Eine durch Migrant/inn/en und/oder ethnische Minderheiten getragene Unternehmenslandschaft wurde als Chance gesehen, die devastierten Zentren der britischen Städte wieder zu beleben. In den von der Depression gekennzeichneten Städten eröffneten bereits in den 1970er Jahren pakistanische und indische Einwanderer zahlreiche kleinere Betriebe. Die britische Regierung unterstützte diese Entwicklung durch spezielle Förderprogramme zusätzlich (BARRET et al. 2000). Bereits Mitte der 1980er Jahre überstieg im UK der Anteil der Selbständigen der asiatischen Minderheit den entsprechenden bei der Bevölkerung ohne Migrationshintergrund (LiGHT 1987, p. 204).

Richtet man den Blick auf die städtische Ebene, so sind zuallererst die klassischen Analysen von SASSEN $(1990,2000)$ zu nennen, wobei diese kein explizites Erklärungsmodell in Bezug auf die Migrantenökonomie konzipierte, sondern diese im breiteren Kontext der Entwicklung in den Global Cities analysierte. SASSEN ortet als Folge der zunehmenden Wanderungsbewegungen ein angebotsseitig wachsendes Potenzial an Immigrant Entrepreneurs. Dazu entsteht in allen Städten nachfrageseitig als Folge der postindustriellen Transformation ein größer werdender Spielraum für kleinunternehmerische Strukturen und zugleich transformieren ethnische Unternehmer/innen die globalen Märkte auf die lokale Ebene. Sie finden in den urbanen Zentren einen fruchtbaren Boden, denn der moderne Lebensstil und veränderte Konsumgewohnheiten der urbanen Mittelklasse verlangen internationale Produkte und verstärken die Externalisierung haushaltsnaher Dienste. Auch die weniger privilegierten Schichten bedienen sich dieser neuen Angebote, da die Familienunternehmen der Migrant/inn/ en - wie der Fast-Food-Sektor zeigt - mit günstiger Preisgestaltung punkten. SASSEN zufolge entstehen neue Gründungschancen daher weniger als oft vermutet in ethnischen Nischen, sondern häufiger in jenen Marktsegmenten, die sich vor allem an den Bedürfnissen der Mehrheitsgesellschaft orientieren, jedoch mangels Lukrativität oder infolge schlechter Arbeitsbedingungen von inländischen Unternehmer/inne/n zunehmend gemieden werden. Dieses Faktum schließt allerdings die zeitgleiche Existenz segregierter Nischenökonomien nicht aus.

\subsection{Die Beziehungen zwischen den kurdischen und türkischen Diasporas in Europa}

In einer globalisierten Welt machen Konflikte an nationalen Grenzen nicht Halt. Auch der türkisch-kurdische Konflikt wurde zu einer transnationalen Konfliktsituation mit entsprechenden Konsequenzen für die Diasporas in den Migrationszielländern. Ein Projekt, welches auf die Entrepreneurship von Kurd/inn/en fokussiert und auf politische Aspekte nicht fokussiert (abgesehen von munizipalen Policies zur Förderung der Diaspora Entrepreneurship), kann die bestehenden politischen und ideologischen Konfliktkonstellationen nicht völlig außer Acht lassen. Sie wurden auch von einem Teil der Interviewten explizit oder implizit angesprochen, andere aber klammerten diesen 
Aspekt als für sie persönlich völlig irrelevant aus. Es kann dennoch nicht Ziel dieser Studie sein, auf die vielschichtigen nationalistischen Konflikte v.a. zwischen Kurd/inn/ en aus der Türkei und der türkischen Diaspora einzugehen. Daher soll nachfolgend nur eine sehr komprimierte Darstellung geboten werden (weiterführend vgl. AmMANN 2001; BASER 17-10-2017; ENGIN 2019; STADLER 2009).

Die Mobilisierung durch politische Organisationen wie die Sozialistische Partei Kurdistans (PSK) sowie die Arbeiterpartei Kurdistans (PKK) verursachte Machtkämpfe mit anderen Diasporagruppen aus der Türkei mit ausgeprägten nationalistischen Tendenzen jeweils auf beiden Seiten. Viele Kurd/inn/en in der Diaspora wollen ihre kurdische Identität, Kultur und Sprache weiter pflegen bzw. nach Jahrzehnten der Repressalien in der Türkei wiederbeleben.

Die Diaspora bietet auch die Möglichkeit der politischen Mobilisierung mit dem Ziel einer Autonomie bzw. Befreiung der Kurd/inn/en, der Anerkennung ihrer Rechte und ethnischen Identität in einigen Herkunftsländern. Seitens mancher türkischer Gruppierungen wird darin ein Indiz für Aktivitäten gesehen, die die nationale und territoriale Integrität der Türkei bedrohen könnten. Insbesondere seit den 1990er Jahren kam es auch in Wien zu Auseinandersetzungen zwischen türkischen und kurdischen Gruppen, die sogar in Angriffen auf Geschäftslokale kurdischer Unternehmer/ innen mündeten und auch in unseren Interviews vielfach angesprochen wurden. Selbst Fußballspiele waren Anlässe für die Austragung der Konflikte. Nationale Symbole wie die türkische Flagge, die PKK-Flagge oder Poster von kurdischen wie türkischen Parteichefs lösten Auseinandersetzungen aus. Wie die interviewten kurdischen Unternehmer/innen das Verhältnis zu den türkischen (und anderen) Communities aus ihrer Perspektive beurteilen, wird im empirischen Teil dieses Berichts anhand von Zitaten aus den Interviews detailliert erläutert.

Im türkisch-kurdischen Konflikt geht es um Identität und die Hegemonie der türkischen über die kurdischen Gruppen. Die türkische Mehrheit versuchte auch in den europäischen Aufnahmeländern immer wieder Hegemonieansprüche über die kurdische Minderheit anzumelden. Aus der Perspektive vieler Kurd/inn/en wird damit die Nichtanerkennung ihrer ethnischen Identität auch außerhalb der Türkei perpetuiert. Aus Sicht mancher Türk/inn/en sympathisieren Staaten der EU mit der kurdischen Diaspora und könnten sogar eine Spaltung der Türkei fördern. Die Integrationsherausforderungen und ökonomischen Krisen in den Aufnahmeländern beider Diaspora-Gruppen trugen eher nicht dazu bei, die Spannungen zu reduzieren. Allerdings beschränken sich offene Auseinandersetzungen ausschließlich auf Teilsegmente beider Gruppen, also solche, die stark politisch mobilisiert sind. Die große Mehrheit der politisch nichtmobilisierten DiasporaMitglieder ist nicht gänzlich frei von Misstrauen (vgl. Erfahrungsbericht Amadia KILIC), doch werden viele Alltagsbeziehungen gepflegt. Vor allem in der Wirtschaft sind Türk/ inn/en und Kurd/inn/en auch als Geschäftspartner/innen tätig oder treten als Kund/inn/ en auf. Die weiteren Entwicklungen der Beziehungen zwischen beiden Gruppen in der Diaspora werden von den künftigen generellen politischen Entwicklungen in der Türkei und der jeweiligen Lage der Kurd/inn/en abhängen. 


\subsection{Relevante Basiskonzepte der Analysen}

\subsubsection{Einleitung}

Da die primäre Zielsetzung unserer Studie in der Analyse empirischer Ergebnisse und nicht in der Voranbringung der Theoriediskussion besteht, soll hier nur ein knapper theoretischer Abriss geboten werden.

Eine Reihe von theoretischen Modellen versucht, die unternehmerischen Aktivitäten von Migranten zu explizieren. Diese sind nicht streng voneinander zu separieren, sondern sie betonen jeweils unterschiedliche Teilaspekte (vgl. KLOOSTERMAN \& RATH 2003, p. 3 ff.; GoldberG \& Sen 1997, p. 57 ff.; NAJib 1994, p. 26 ff.; IKonomu 1989, p. 313 ff.; Jenkins 1984, p. 231 ff.; Jones et al. 2014) des Phänomens der Migrantenselbständigkeit, die auch typisch sind für bestimmte Phasen der Migration. In den „fortgeschritteneren“ Zuwanderungsgesellschaften Europas setzte das wissenschaftliche Interesse am Business von Migrant/inn/en in den späten 1980er Jahren ein. Mit der Herausgabe von „Ethnic Communities in Business“ durch WARD \& JENKIns (1984) erfolgte erstmals eine Zusammenschau der bis dahin verfügbaren Forschungsergebnisse und wurde der erste Grundstein für eine systematische Befassung mit ethnischen Ökonomien nicht nur in Großbritannien, sondern auch in Kontinentaleuropa gelegt. Als weiterer "Klassiker" auf dem Gebiet der ethnischen Ökonomie ist die Analyse von WALDINGER et al. (1990a, b, c) anzuführen (zwecks Überblicks vgl. auch LiGHT \& Gold 2000; Kloosterman \& RATH 2003; Dana 2007; Dana et al. 2008). Deren theoretisch-analytische Zugänge determinieren nach wie vor in einem erheblichen Ausmaß die sozialwissenschaftliche Auseinandersetzung mit dieser Thematik, obwohl die darin enthaltenen Modelle inzwischen ergänzt und weiterentwickelt wurden.

\subsubsection{Das Konzept der ,mixed embeddedness ${ }^{66}$}

Ein weiteres relevantes Konzept zur Untersuchung des Unternehmertums von Immigrant/inn/en ist das der ,, mixed embeddedness “ (JONES et al. 2014; KLOOSTERMAN, 2010; Kloosterman et al. 1998, 1999; Price \& CHACKo, 2009). Dieses bezieht sich auf die Einbettung von Immigrant/inn/en in soziale Netzwerke von Diaspora-Communities und in das sozioökonomische und politisch-institutionelle Umfeld der Aufnahmegesellschaft. KLOOSTERMAN \& RATH $(2001,2003)$ verknüpften das Konzept von WaLDINGER et al. (1990c) mit jenem der drei grundlegenden wohlfahrtsstaatlichen Modelle von ESPING-ANDERSEN (1990) und rückten die Einbettung unternehmerischer Aktivitäten in unterschiedliche soziale Kontexte sowie sozialstaatliche Regulationen von Selbständigkeit in den Mittelpunkt ihres Modells. Es setzt an den Wechselwirkungen zwischen den Ressourcen der Unternehmer/innen mit Migrationshintergrund, den urbanen und nationalen Strukturen sowie den diese determinierenden Institutionen an.

Unbestritten ist die Relevanz des ethnisch-kulturellen Faktors und der sozioökonomischen Positionierung der einzelnen Migrantengruppen. Dies betrifft Fragen des 
Bildungssystems im Herkunftsland und des Bildungsniveaus der Immigrant/inn/en (vgl. Goldberg \& Sen 1992, 1997). Zu berücksichtigen ist auch, welche Bedeutung die unternehmerische Selbständigkeit als Alternative im Erwerbsverhalten besitzt und des Weiteren die generelle Wertschätzung der Unternehmerrolle (LiGHT et al. 1990). Die Zukunftspläne für die (ursprünglich geplante) Rückkehr vieler türkischer Migrant/ inn/en beispielsweise waren traditionellerweise primär auf eine selbständige Tätigkeit ausgerichtet. Mit der Verfestigung des Aufenthalts in den Migrationszielländern und der Abschwächung der Rückkehrorientierung verwirklichten zahlreiche Migrant/inn/ en aus der Türkei ihren Traum vom Unternehmer/innen/tum schließlich im jeweiligen Zielland der Migration. Zuwander/er/innen sind daher aufgrund dieser divergierenden Voraussetzungen und des zeitlichen Rahmens der Migration unterschiedlich von (des) integrationspolitischen Maßnahmen der Aufnahmegesellschaft betroffen (vgl. KLOosTERMAN \& RATH 2003).

Ein Ziel des Embeddedness-Konzepts ist es, soziale Beziehungen und Interaktionsstrukturen (,social networks") in Handlungs- und Entscheidungskontexten stärker zu gewichten (vgl. auch PORTES \& SENSENBRENNER 1993): „Actors do not behave or decide as atoms outside a social context, nor do they adhere slavishly to a script written for them by the particular intersection of social categories that they happen to occupy. Their attempts at purposive action are instead embedded in concrete, ongoing systems of social relations “ (GRANOVETTER 1985, p. 485). Hierbei differenzierte GRANOVETTER auch zwischen relationaler und struktureller Einbettung. Erstgenannte meint die direkten persönlichen Beziehungen zwischen ökonomischen Akteur/inn/en. Strukturelle Einbettung bezieht sich auf das Netzwerk sozialer Beziehungen ökonomischer Akteur/ inn/e/n (z.B. Immigranten-Communities) und beeinflusst ökonomisches Handeln subtiler als die relationale Einbettung (vgl. ibid. 1990, p. 98 f.). Die Embeddedness in eine Diaspora fördert die Entrepreneurship durch den Zugang zu sozialen Netzwerken, die Finanzkapital, diverse Unterstützungsleistungen, Knowhow, eine Angebotsstruktur sowie einen bestimmten Kund/inn/enstamm (TAVASSOLI \& TRIPPL 2017) bereitstellen. Diese Netzwerke sind für Immigrant/inn/en besonders wichtig, da diese im Vergleich zu Einheimischen weniger Wissen über lokale Richtlinien, soziale und unternehmerische Normen, kulturelle Routinen etc. aufweisen. Die Stärke des Einbettungskonzepts besteht darin, dass es die sozialen Strukturen, in die ökonomische Handlungs- und Entscheidungsprozesse eingebettet sind, als wesentlichen Faktor berücksichtigt.

Eine unternehmerische Aktivität setzt voraus, dass Personen zum einen eine individuelle Entscheidung zum Schritt in die Selbständigkeit treffen und zum anderen sind es strukturelle Bedingungen bzw. das Mikro- und Makroumfeld, welche diese Entscheidungsfindung beeinflussen (Ziegler \& Hinz 1992; Welter \& Rosenbladt 1998). Einen wichtigen Aspekt für die strukturellen Bedingungen auf der Makroebene bildet auch im Rahmen der „mixed embeddedness“ das Bedingungsgeflecht der „opportunity structures". ${ }^{3}$ Es berücksichtigt den politischen und ökonomischen Kontext, der die Rahmenbedingungen für unternehmerische Tätigkeiten absteckt. Als Teil-

3 Vgl. dazu Kloosterman \& Rath (2003), Rath (2002), Portes \& Sensenbrenner (1993). 
elemente der Opportunity Structures werden sozialräumliche, verwaltungsbezogene und selbstverständlich ökonomische Strukturkonstellationen von den Selbständigen mit Migrationshintergrund genutzt. Die Opportunity Structures umfassen steuerrechtliche Bestimmungen, Gesundheits- und Sicherheitsbestimmungen, Bestimmungen der Bauordnung und Flächenwidmung, Unternehmensförderungsmaßnahmen, die Vergabe von (Mikro)krediten, Institutionen, die die Einhaltung der relevanten Regeln kontrollieren sowie das bestehende Regelwerk per se. Das Unternehmertum von Migrant/ inn/en ist in sozial, informell und ökonomisch vernetzte Strukturen eingebettet, womit politische und wirtschaftliche Institutionen sowie Regulative als grundlegend relevant für das Verstehen und die Analyse dieses Teils der Ökonomie angesehen werden. Als Wissenschaftler/innen an einem raumwissenschaftlich-geographisch ausgerichteten Institut sind wir unter anderem auch an den räumlichen Aspekten der Opportunitätsstrukturen interessiert.

Es wird damit von Wechselwirkungen zwischen den Migrant/inn/en und den im Zuwanderungsland vorhandenen Rahmenbedingungen ausgegangen. Migrant/inn/en reagieren nicht nur auf vorhandene Bedingungskonstellationen, sondern sind sehr wohl auch imstande, solche Bedingungen durch ihr Tun aktiv zu beeinflussen. KLOOSTERMAN \& RATH (2001) betonten die aktive Rolle und die Gestaltungsspielräume, die unternehmerische Tätigkeit den Migranten bietet. Die Migrantenökonomie ermöglicht es den Zuwanderer/inne/n, ihre eigenen Arbeits- und Lebensweisen in einem höheren Ausmaß $\mathrm{zu}$ bestimmen und zudem den räumlichen Kontext der Stadt zu beeinflussen und zu gestalten (vgl. HiLlmann 2007; Hillmann \& Sommer 2011).

Entwicklungen im Kontext der Migrant Economy spiegeln Dynamiken und Veränderungsprozesse wider, die die gesamte urbane Ökonomie betreffen. Beispiele dafür sind etwa die Zunahme der beruflichen Selbständigkeit angesichts eines Rückgangs der unselbständigen Vollzeitarbeitsverhältnisse sowie eines generellen Prekarisierungstrends auf dem Arbeitsmarkt oder die steigenden Insolvenzzahlen in wirtschaftlichen Krisenphasen. Unternehmerische Opportunitätsstrukturen befinden sich nicht in einem institutionellen Vakuum, sondern sind eingebettet in institutionelle Rahmenbedingungen. Dass die institutionellen Rahmenbedingungen als Determinante der Entwicklung migrantischen Unternehmertums von immenser Bedeutung sind, hat KLOOSTERMAN (2000) aus theoretischer Perspektive analysiert. Märkte, auch weitgehend liberalisierte, werden stets auf die eine oder andere Weise reguliert. Diese Regulierungen reichen von PositiveAction-Maßnahmen bis zu umfassenden Wirtschaftsförderungsprogrammen.

Die Opportunity Structures repräsentieren einen analytischen Schlüsselfaktor. Da sich urbane Ökonomien aktuell in einem permanenten Wandlungsprozess befinden, sehen sich auch Unternehmer/innen mit Migrationshintergrund mit kontinuierlich wandelnden Opportunity Structures konfrontiert, auf die sie, um erfolgreich zu bleiben (oder einfach um zu überleben), flexibel reagieren müssen. Following the "mixed embeddedness' logic, [...] various components of the urban economy interact to produce a complex but also dynamic ecological system, dramatically affecting the political economy of cities 
and, in so doing, entrepreneurial opportunities" (RATH \& SWAGERMAn 2011, p. 11). Die Aspekte der Opportunitätsstrukturen werden in vielen Studien zwar angesprochen, jedoch zumeist nicht im Detail analysiert, Städtevergleiche fehlen größtenteils.

Weiters darf nicht übersehen werden, dass ähnliche strukturelle Rahmenbedingungen auf unterschiedliche Migrantengruppen und deren Potenziale unterschiedliche Auswirkungen haben. Nach Hillmann \& Sommer (2011, p. 37) sind diese „,Unterschiede nur zu einem geringen Teil auf die kulturellen Eigenschaften einzelner Nationalitätengruppen zurückzuführen, sondern müssen unter Berücksichtigung der Migrationsgeschichte interpretiert werden ". So spielen einerseits die deutlich unterschiedlichen Qualifikationsstrukturen eine erhebliche Rolle. Die Gastarbeiter waren zumeist unge-lernte Arbeitskräfte, bei der Arbeitskräftezuwanderung aus Ost(mittel)europa handelt es sich vor allem um Qualifizierte. Dieses Faktum spielt bei der Beantwortung unserer zentralen Forschungsfrage, warum es unterschiedlichen Herkunftsgruppen in Wien die Präsenz selbständiger Erwerbstätigkeit so stark differiert, eine entscheidende Rolle. Dass der ethnische Faktor dennoch eine gewisse Rolle spielt, belegen die Analysen von Lamadem (1995), Welter (2005), Pütz et al. (2007), SAhin et al. (2007), WANG (2008) und YAVUZCAN (2003).

Andererseits prägen auch politische und legistische Rahmenbedingungen die Opportunitätsstrukturen und somit die selbständige Erwerbstätigkeit von Zuwanderern entscheidend. Die EU-Erweiterungen hatten beispielsweise abrupte Veränderungen des Rechtsstatus von Bürger/inne/n aus den neuen Beitrittsländern in Ost- und Südosteuropa zur Folge, was sich auch auf die Unternehmer/innen unter ihnen auswirkte. Im Gegensatz zu Großbritannien, Italien, Griechenland oder Portugal existier(t)en in vielen mittel- und nordeuropäischen Staaten rigide rechtliche und faktische Zugangsbarrieren zur selbständigen Erwerbstätigkeit für Migrant/inn/en. Diese strengeren rechtlichen Rahmenbedingungen führten in Wien im Vergleich zu britischen und niederländischen Metropolen zu einem späteren Einsetzen des Trends zum Unternehmertum bei Immigrant/inn/en. HABERFELLNER (2000, et al. 2000) stellte des Weiteren für Österreich in ihrer Analyse fest, dass das strikte Regulierungskorsett eine wesentliche Hürde für die Entfaltung der Unternehmen von Migrant/inn/en darstellt.

\subsubsection{Soziale Netzwerke - Granovetter „Weak and Strong Ties“}

\subsubsection{Einbettung in Netzwerke der Diaspora-Communities}

Die Neue Wirtschaftssoziologie GranovetTERs etablierte sich als Gegenpol zum individualistischen Handlungsmodell der klassischen Ökonomie (GRANOVETTER \& SwEDBERG 2011, p. 6), indem GRANOVETTER der Neoklassik eine „untersozialisierte“ und somit realitätsfremde Theorie menschlichen Handelns vorwarf. Dieses Konzept bietet eine optimale Basis für die Analyse unternehmerischen Handelns in sozialen Interaktionsgeflechten, indem mit der Fokussierung auf die handlungsbezogene Ebene 
sozialer Einbettung der Bezug zu generelleren gesellschaftlichen Zusammenhängen zu kurz kommt (vgl. Barrett et al. 2000; Neckel 2008; Deutschmann 2007). Das Konzept von GranovetTER (1985) fokussierte auf die soziale Einbettung ökonomischen Handelns in modernen Gesellschaften: "By, embeddedness 'I mean that economic action, outcomes and institutions are affected by actors ' personal relations, and by the structure of the overall network of relations " (GRANOVETTER 1990, p. 98).

Basierend auf GranovetTERs (1985) Konzept der Relevanz der Einbettung von wirtschaftlichen Aktivitäten in soziale Netzwerke diskutierte Williamson $(1991,1996)$ Netzwerke als drittes wesentliches organisatorisches Konstrukt für das Unternehmertum neben Marktmechanismen und der vertikalen Integration. Dabei sind aus netzwerkanalytischer Perspektive zwei Hauptkategorien - formelle und informelle Netzwerke (JohanNisSON 1986) - zu unterscheiden. Formelle Netzwerke basieren auf Beziehungen auf Firmen- und Geschäftsebene, während informelle Netzwerke aus Beziehungen auf individueller und privater Ebene bestehen. Weitere Klassifikationen beziehen sich auf interorganisatorische und soziale Netzwerke (BROWN \& BUTLER 1993) von Unternehmer/inne/n (O`Donnel et al. 2001). Die potenziellen Vorteile, die Unternehmer/innen aus ihren Netzwerken ziehen können, sind vielfältig, etwa (a) die Identifikation und Nutzung von Chancen, (b) der Zugang zu Ressourcen und (c) die Stimulation unternehmerischer Motivation. Hinsichtlich unternehmerischer Chancen sind die Analysen von Kontinen \& OJala (2011); Stuart \& Sorenson (2007); Arenius \& De Clercq (2005) sowie Hansen (1995) anzuführen. Hansen (1995) betont, dass Netzwerke Produkt- und Dienstleistungsideen entstehen lassen, welche für die unternehmerische Genese und die Wahrung von Business-Chancen wichtig sind. Außerdem können Unternehmer/innen auf Basis von Netzwerken auf vielfältige Ressourcen zugreifen, wie z.B. Wissen (Stuart \& Sorenson 2007), die Rekrutierung von Fachkräften (BIrLey 1985; STUART \& SORENSON 2007), den Zugang zu Finanzkapital (Fried \& HisRich 1994; HsU 2004) und spezifische Branchenkenntnisse (BERGMAN et al. 2004). STUART \& SORENSON (2007) argumentieren, dass soziale Netzwerke unternehmerische Prozesse deshalb beeinflussen, weil sie die privaten Informationskanäle bereitstellen, die für die Identifizierung von Chancen relevant sind. Netzwerke können auch das Überleben von Firmen im harten unternehmerischen Wettbewerb fördern, indem sie Unternehmer/innen motivieren, nötige Veränderungen umzusetzen oder das Wachstum des Unternehmens zum richtigen Zeitpunkt zu forcieren (TJOSVOLD \& WEICKER 1993). Letzere hoben vor allem die kooperativen Beziehungen innerhalb sozialer Netzwerke hervor. Diese wirken sich positiv auf die Lebensdauer von Unternehmen aus, indem sie die Motivation, emotionale Unterstützung und das Selbstvertrauen fördern.

Speziell die Diaspora-Netzwerke können Unternehmer/inne/n auch zum Zugang zu institutionellen und marktrelevanten Informationen (FREINKMAN 2011) sowie zu speziellen branchenbezogenen Kenntnissen (TALIB et al. 2012; SAXenian 2001) verhelfen. Die Diaspora-Netzwerke sind es, die Menschen mit demselben ethnischen Hintergrund verbinden. Sie können auch als ein sozialer Pool und Stimulus für ihre unternehmerischen Aktivitäten fungieren (KuZnetsov 2006; HARIMA 2014). Die 
Informationsflüsse innerhalb der Diaspora-Communities kompensieren den Mangel an Ressourcen oder Informationen in anderen Bereichen und verhelfen den Diasporas dazu, kulturelle und institutionelle Barrieren zu überwinden (NANDA \& KHANNA 2007). Dies spielt eine besonders große Rolle in jenen Diaspora-Communities bzw. bei Angehörigen derselben, die Probleme mit der Beherrschung der Sprachen der Aufnahmeländer, den komplizierten rechtlichen und institutionellen Rahmenbedingungen sowie einen Mangel an verfügbaren Ressourcen haben. Die Diaspora als ethnische Enklave ermöglicht es auch unternehmensrelevante Personen wie Lieferant/inn/en, Kund/inn/en und Mitarbeiter/innen zu finden (SALAFF et al. 2003). Neben dem Zugang zu Wissen und Humanressourcen ermöglicht das Diaspora-Netzwerk auch das Auffinden möglicher Investor/ inn/en. Oft investieren Diaspora-Angehörige eher in die Unternehmen von Menschen mit demselben ethnischen Background, wobei auch verwandtschaftliche Beziehungen sowie das Ziel eine Rolle spielen kann, die Situation im jeweiligen Herkunftsstaat zu verbessern (GILLESPIE et al. 1999). Es ist typisch, dass Diaspora-Unternehmer/innen ihr Grundkapital weniger auf Basis von Bankdarlehen, sondern oft durch Familienmitglieder oder Freund/inn/e/n aufbringen (BoyD 1989). Diese garantieren mehr finanzielle Flexibilität als Kreditinstitute, denn sie sind in Krisenzeiten bereit, fällige Kreditrückzahlungen zu stunden oder weitere Darlehen zu gewähren. Trotz der Relevanz von Diaspora-Netzwerken als wichtige Ressource (KUZNETSOV 2006; SAXENIAN 2001; Dutia 2012) wurde den Einflüssen dieser Netzwerke auf den Prozess der Identifikation von Unternehmenschancen sowie auf die Genese und den Wandel von Motivationen für die Entrepreneurship bislang nur wenig Augenmerk geschenkt.

\subsubsection{Genderspezifische Netzwerkstrukturen}

Hinsichtlich der genderspezifischen Determinanten kommt neuerlich das Konzept Granovetters $(1973,1985,1990)$ ins Spiel. Dieses belegt bezüglich der Netzwerkstrukturen, dass Frauen im Vergleich zu Männern eher „strong ties“ eingehen, d.h. engere persönliche Beziehungen, anstatt der stärker opportunistisch motivierten und emotional distanzierteren ,weak ties“, die vor allem Männer präferieren (vgl. Воотн 1972; Moore 1990; Benenson 1993; Baumeister \& Sommer 1997). Frauen bauen tendenziell häufiger auf Netzwerke, die stabiler, emotionaler und pfadabhängiger sind und ihre Stabilität und Tragfähigkeit aus einer Dominanz der „strong ties“ gegenüber den weniger belastbaren und solidarischen ,weak ties“ ableiten. Frauen neigen auch dazu, ihre informellen Netzwerke viel stärker innerhalb ihres (erweiterten) Familien- und Freundeskreises aufzubauen (vgl. IBARRA 1992; BRUSH 1992; MUNCH et al. 1997; VerHeUL et al. 2002). Sie agieren sozial selektiver und investieren weniger Zeit und Aufwand in flüchtige Kontakte.

Männer sind hinsichtlich der Gestaltung ihrer sozialen Netze weniger selektiv und handeln in summa ausgeprägter zweckrational in ihrem zwischenmenschlichen Verhalten. Dies führt zur Entwicklung von Netzwerken mit größeren Zahlen an immer wieder neuen und variierenden Bindungen basierend vor allem auf „weak ties“. Männer verlassen sich im Gegensatz zu Frauen mehr auf formelle und lockere infor- 
melle Netzwerke. Formelle Kontakte sind es aber, die im Vergleich zu „strong ties“ effizientere berufsbezogene Vorteile begünstigen und die gerade bei der Gründung eines Unternehmens relevante und nützliche Informationsquellen sein können. GRANOVETTER $(1973,1985,1990)$ wies nach, dass eher zufällige und opportunistische Kontakte in Kontexten wie der Arbeitssuche nützlicher sind, da hierbei die Fähigkeit von Kontaktpersonen, neue Informationen zu liefern, ihre geringere Motivation, Unterstützung und Hilfe kompensiert. Diese Unterschiede üben einen erheblichen Einfluss auf die unternehmerischen Motivationen, Gründungsentscheidungen und die unternehmerische Effektivität von Frauen aus. Basierend auf empirischer Evidenz kann also argumentiert werden, dass sich die Determinanten der Unternehmensgründung bei Immigrant/inn/en nach der Geschlechtszugehörigkeit unterscheiden können (vgl. CoLOMBELLI et al. 2019; Lindenlaub \& Prummer 2014; Lalanne \& Seabright 2014).

Colombelli et al. (2019) fanden empirische Evidenz dafür, dass der Bestand an Migrant/inn/enunternehmen eine größere Zahl weiterer Unternehmen der gleichen Community im selben Sektor und auch geographisch - in derselben Provinz - induziert und dass dieser Effekt bei Männern ausgeprägter ist als bei Frauen. Außerdem stellten dieselben Autor/inn/en fest, dass die Geschlechterunterschiede bei Immigrant/inn/en aus Herkunftsländern mit allgemein geringer ausgeprägter ,gender equality“ ausgeprägter sind.

Männer und Frauen unterscheiden sich auch hinsichtlich der Nutzung von sozialen Netzwerken. Etliche Arbeiten (MunCH et al. 1997; Aldrich 1989; Cromie \& BiRLEY 1992; HANSON \& BLAKE 2009) zeigen, dass Unternehmerinnen im Vergleich zu ihren männlichen Kollegen insgesamt weniger Netzwerkkontakte pflegen. Die hauptsächliche Begründung dafür ist, dass Frauen ihre unternehmerische Laufbahn typischerweise aus häuslichen oder nichtleitenden Positionen heraus beginnen und ihr Ausgangsnetzwerk daher weniger umfangreich und entwickelt ist. Hinzu kommt, dass Unternehmerinnen auch weniger Zeit in den Aufbau von Netzwerken investieren können, da sie im Vergleich zu männlichen Gründern mit höheren Barrieren hinsichtlich der Vereinbarkeit von Familie und Beruf konfrontiert sind (Verheul et al. 2002). Darüber hinaus besteht Evidenz dafür, dass die Geschlechter ihre Netzwerke unterschiedlich nutzen (MENGEL, 2015; BEAMAN et al. 2016) und aufgrund divergierender Netzwerkstrukturen unterschiedliche unternehmensbezogene Vorteile erzielen (Lindenlaub \& PRUMmer, 2014; Lalanne \& Seabright, 2014). Unterschiede in den sozialen Netzwerken von Männern und Frauen können daher ein Schlüssel zum Verständnis von geschlechtsspezifischen Unterschieden in den Karrierepfaden und -erfolgen sein und könnten Erklärungen für die anhaltenden geschlechtsspezifischen Unterschiede hinsichtlich der Arbeitsmarktpositionierungen bieten (BERTRAND 2011).

\subsubsection{Role Models}

Genderbezogene Unterschiede hinsichtlich der Entrepreneurship sind nicht allein auf der Basis wirtschaftlicher Entwicklungen zu erklären, sondern sozioökonomische 
Variablen, die Entwicklung der Geschlechterrollenkonzepte und der sozioökonomische Status spielen ebenfalls eine Rolle. ManiYalath \& NARENDRAN (2016) zeigten in ihrer empirischen Analyse unter Verwendung von GEM-Daten zu 61 Ländern, dass nationale Indizes auf Basis geschlechtsspezifischer Variablen einen Einfluss auf die Unternehmerinnenquote haben. Es sind auch geschlechtsspezifische Institutionen, die einen relevanten Einfluss auf die Entscheidung von Frauen, ein eigenes Unternehmen zu gründen, ausüben (Elam \& TerJesen 2010; PathaK et al. 2013; Urbano \& Alvarez 2014). Gemäß den genannten Autor/inn/en spielen zwei Indizes zu den Gender Role Models als wesentliche Determinanten für weibliches Unternehmertum eine Rolle: 1) Empowerment (gemessen am Anteil der von Frauen besetzten Parlamentssitze und am Anteil erwachsener Frauen mit zumindest Sekundarschulbildung) und 2) ökonomischer Status (d.h. Arbeitsmarktbeteiligung). Diese Faktoren spielen auch hinsichtlich der Struktur sozialer Netzwerke eine Rolle. Je krasser die Statusungleichheit der Geschlechter desto größer sind die Unterschiede bezüglich der Intensität und Gestalt sozialer Netze zwischen Männern und Frauen (Colombelli et al. 2019). Demgemäß ist davon auszugehen, dass in weniger gleichberechtigten Gesellschaften, in denen die Arbeitsmarktbeteiligung und das Bildungsniveau von Frauen niedrig sind, eher informelle, geringer fomalisierte und weniger tragfähige Netzwerkaktivitäten von Frauen vorhanden sind. Das geringere Level des Empowerments und der schwächere ökonomische Status von Frauen in weniger gleichberechtigten Gemeinschaften sind Indikatoren für einen Mangel an unternehmerischen Role Models innerhalb der jeweiligen Communities, in denen Frauen eine etwaige unternehmerische Karriere typischerweise in haushaltsbezogenen bzw. nichtleitenden Positionen starten.

\subsubsection{Communityspezifische Effekte}

Obwohl noch immer zu wenig Daten über die treibenden Kräfte des Unternehmertums von Immigrant/inn/en vorhanden sind, steht außer Diskussion, dass bestimmte Communities dazu neigen, sich in ganz bestimmten Geschäftsbereichen zu konzentrieren. Diaspora-Communities können also per se durch Rollenvorbilder die Entrepreneurship fördern (KLOOSTERMAn et al. 1999; Light et al. 1993; RodRigueZPose \& VON BerlePsCh, 2014; TAVASSOli \& TRIPPl 2017). Durch einen kombinierten Lern- und Nachahmungseffekt werden Personen in Diasporas von Entrepreneurs in derselben Community beeinflusst. Merkmale wie Branche und die Zugehörigkeit zu einer Diaspora-Community fördern die Rollenidentifikation von Unternehmer/inne/n. KERR \& MANDORFF (2015) dokumentierten dieses Phänomen in den Vereinigten Staaten und erklärten es durch ein Modell, das aufzeigt wie soziale Beziehungen in (Teil-) Märkten langfristige berufliche Stratifizierung erzeugen können. TAVASSOLI \& TRIPPL (2017) untersuchten den Einfluss ethnischer Communities auf die Entrepreneurship von Immigrant/inn/en in Schweden und stellen fest, dass Zuwander/er/innen eine höhere Neigung zeigen, sich als Unternehmer/in selbständig zu machen, wenn sie in lokalen Kontexten leben, die einen hohen Anteil an Unternehmer/inne/n derselben Ethnie und derselben Wirtschaftssektoren und -branchen aufweisen. Sie erklären dies als Ergebnis 
von Communities, die als Anbieter von branchenspezifischem institutionellem Wissen fungieren, und durch den ,Role Model“"-Effekt (Bosma et al. 2012; CHLOSTA et al. 2012).

\subsubsection{Der Gender Gap in der Migrant Entrepreneurship}

Obwohl nicht wenige Studien die Existenz eines Gender Gap in der Migrant Entrepreneurship dokumentierten, gab es bislang nur selten breitere empirische Untersuchungen zur Relevanz der Gendervariable für die unternehmerische Tätigkeit (vgl. etwa BRIEGER \& GIELNIK 2021). Die Forschung war viel stärker auf männliche Immigranten als Unternehmer fokussiert, während die Entrepreneurship von Frauen mit Migrationshintergrund eher vernachlässigt wurde (Collins \& Low 2010; MARCHAND \& SiEGEL 2014). Viele individuelle und kontextuelle Faktoren, welche die geschlechtsspezifischen Unterschiede bedingen, sind noch kaum systematisch untersucht. Generell wurde festgestellt, dass zugewanderte Frauen größere Hindernisse im Vergleich zu ihren männlichen Landsleuten vorfinden (VACCARINO et al. 2011; AZMAT 2013).

Als Ausgangsbasis dient dieser Studie das Konzept der Mixed Embeddedness, das Aspekte der wirtschaftlichen, sozialen und politisch-institutionellen Rahmenbedingungen des Aufnahmelandes integriert. Es bietet somit einen adäquaten Rahmen für die Interpretation jener Determinanten, welche die Entrepreneurship fördern (KLOosTERman et al. 1999; RATH \& Kloosterman 2000; Kloosterman \& Rath 2001; KloosTERMAN 2003; WANG \& WARN 2017). Methodisch gestattet es, die Effekte in Bezug auf die ökonomische Situation im Aufnahmeland (Marktchancen und Marktzugang), soziale Netzwerke, migrationspolitische Rahmenbedingungen und institutionelle Faktoren (unternehmensfreundliche Regulierungen, institutioneller Support, Rechtsstaatlichkeit) auf die geschlechtsspezifischen Unterschiede in der Diaspora Entrepreneurship zu untersuchen.

Die zentrale Ausgangshypothese ist, dass ein der Selbständigkeit förderliches Umfeld die Beziehungen zwischen der Variable Geschlecht und der Entrepreneurship in der kurdischen Community in einer Weise beeinflusst, von der besonders die Frauen profitieren.

In Orientierung an VACCARINO et al. (2011) und AZMAT (2013) argumentiert diese Studie, dass sich immigrierte Frauen in einer benachteiligten Position befinden und dass sie daher stärker auf ein unterstützendes Umfeld (persönliche Netzwerke, institutionelle Beratung, finanzielle Unterstützung in Form von Förderungen etc.) angewiesen sind. Personen, die als Geflüchtete nach Österreich kamen, sind tendenziell noch vulnerabler und haben weniger Zugang zu den nötigen Ressourcen. Dementsprechend ist zu antizipieren, dass ein supportives Umfeld für Asylberechtigte noch wichtiger ist als für Arbeitsmigrant/inn/en, die sich selbständig machen wollen.

Das vorliegende Projekt möchte zur Debatte über die Diaspora-Entrepreneurship in zweierlei Hinsicht beitragen. Erstens, indem ein interaktionistischer Ansatz verfolgt wird (vgl. Frese \& GIELNIK 2014; WeLter 2010) soll analysiert werden, wie ein sozial und institutionell unterstützendes Umfeld die unternehmerische Aktivität von Kurdinnen 
und Kurden fördert. Zweitens wird eine kontextuelle Perspektive gewählt, um zu einem besseren Verständnis der geschlechtsspezifischen Unterschiede im Unternehmertum von Kurd/inn/en in Wien beizutragen (vgl. BRIEGER \& GIELNIK 2021).

Untersuchungen zeigen, dass Frauen im Allgemeinen weniger häufig unternehmerisch tätig sind als Männer (Kelley et al. 2015; MinNiti \& NAUdÉ 2010). Dieser Gender Gap ist auf individuelle und kontextuelle Faktoren, wie z.B. soziodemographische Merkmale, unternehmerische Einstellungen, familiärer Hintergrund und Umweltbedingungen (KLYVER et al. 2013; Kelley et al. 2015), zurückzuführen. In Bezug auf die individuelle Ebene zeigt sich, dass die Selbsteinschätzungen von Frauen über die notwendigen Fähigkeiten zur Gründung und Führung eines Unternehmens geringer sind als jene von Männern (Koellinger et al. 2013; WiLson et al. 2007). Dieses niedrigere Niveau der Überzeugung von eigenen unternehmerischen Kompetenzen unter Frauen ist ein globales Phänomen und nicht auf bestimmte kulturelle Kontexte beschränkt (WiLsON et al. 2007). Nach THÉBAud (2010) ist der Anteil der Frauen, die meinen über Fähigkeiten und Erfahrungen zum Unternehmertum zu verfügen, um 50\% niedriger als bei Männern. Darüber hinaus sind ihre Ängste vor dem Scheitern erheblich stärker ausgeprägt, während ihre Risikobereitschaft gering ist (BöNTE \& PIEGELER 2013; Caliendo et al. 2009). Muravyev et al. (2009) und Fairlie \& RobB (2009) identifizierten den eingeschränkten Zugang von Frauen zu finanziellen Ressourcen als einen wesentlichen Grund für den Gender Gap. Darüber hinaus weisen Frauen weniger und andere soziale Netzwerke auf (Verheul \& THuriK 2001; Koellinger et al. 2013). Die schwächeren finanziellen und sozialen Ressourcen erklären auch, weshalb unternehmerische Neugründungen von Frauen auf dem Markt geringere Überlebenschancen haben (VAN DER ZWAN et al. 2012).

Nach BRIEGER \& GielniK (2021) gelten die genannten Faktoren auch bei der Entrepreneurship in Diaspora-Communities, wobei hier weitere Faktoren hinzukommen. Immigrant/inn/en werden mit größeren Hindernissen bei der Beschaffung von Start-upKapital sowie bei der Entwicklung von formellen und informellen sozialen Netzwerken konfrontiert (Vaccarino et al. 2011; Azmat 2013). Darüber hinaus haben Migrantinnen (analog zu Frauen ohne Migrationserfahrung) eher eine ins Negative verzerrte Wahrnehmung eigener Kenntnisse und Fähigkeiten, zeigen eine größere Risikoaversion und haben möglicherweise weniger Zugang zu den zur Gründung und Führung eines Unternehmens relevanten Ressourcen, was die Wahrscheinlichkeit sich selbständig zu machen, weiter reduziert (TERJESEN et al. 2016). Der Gender Gap hinsichtlich unternehmerischer Tätigkeiten dürfte bei ihnen noch stärker sein.

Studien zeigen, dass der Kontext die geschlechtsspezifischen Unterschiede hinsichtlich der Entrepreneurship determiniert. In höher entwickelten Volkswirtschaften sind die geschlechtsspezifischen Unterschiede der unternehmerischen Aktivitäten größer (KLYVER et al. 2013; VAN DER ZWAN et al. 2012), da in diesen unterstützenden Institutionen für Frauen vorhanden sind, die ökonomische Zwänge in Richtung Selbständigkeit neutralisieren. Des Weiteren sind kulturelle und institutionelle Faktoren relevant für den Gender Gap (BAughn et al. 2006; Mueller 2004; Shinnar et al. 
2012; KLYver et al. 2013). Gemäß KLYver et al. (2013) bedeutet ein höheres Niveau der Geschlechtergleichheit in den Sektoren Wirtschaft, Bildung, Politik und Gesundheit, dass der Gender Gap der Entrepreneurship größer ist. Laut THÉBAUD (2015) müssen Frauen die Selbständigkeit nicht als Ausweichstrategie wählen, wenn institutionelle Angebote der Kinderbetreuung oder attraktive Teilzeitbeschäftigungsmöglichkeiten vorhanden sind.

In den Diaspora-Communities ist die Entrepreneurship entweder Ausweichstrategie oder ein bewusst gewählter Weg. Die Faktoren geringes Humankapital, unzureichende Kenntnis der Landessprache, niedrige Bildungsabschlüsse und wenig Berufserfahrung beeinflussen die Suche nach einer unselbständigen Beschäftigung (RATH \& Kloosterman 2000; Collins \& Low 2010). Selbst Frauen mit guter Bildung und beruflicher Qualifikation müssen zur Kenntnis nehmen, dass ihre Fähigkeiten im Aufnahmeland weniger anerkannt werden. Die Chancen auf eine unselbständige Beschäftigung sind damit deutlich reduziert (Levie 2007; Collins \& Low 2010). Damit wird die Entrepreneurship für manche zu einer attraktiven Alternative zur Finanzierung des Lebensunterhalts. Als Selbständige haben sie eher die Möglichkeit ihre vorhandenen Qualifikationen und Erfahrungen einzubringen und Einkommen zu generieren. Nach BRIEGER \& GIELNIK (2021) scheint der Grenznutzen von unterstützenden Rahmenbedingungen für Frauen sogar höher zu sein als für Männer. Dies liegt daran, dass Frauen aus DiasporaCommunities meist im Vergleich zu Männern ein geringeres Level an unternehmerischem Selbstvertrauen besitzen und geringere finanzielle und soziale Ressourcen haben. Somit ist der Vorteil, den sie aus unterstützenden Kontexten ziehen, größer. Mittels Förderungen jeglicher Art können Frauen mit Migrations- oder Fluchthintergrund den Mangel an Selbstvertrauen und Ressourcen besser kompensieren, was zu einer Verringerung des Gender Gaps beiträgt.

Außer Frage steht, dass soziale Netzwerke einen beträchtlichen Einfluss auf unternehmerische Aktivitäten ausüben (KLYVER \& FOLEY 2012; STAM et al. 2014). Dies ist eine Kernargumentation des Mixed-Embeddedness-Ansatzes, der die Relevanz der Einbettung in Netzwerke sowohl innerhalb der Diaspora-Communities als auch mit der Aufnahmegesellschaft betont (RATH \& Kloosterman 2000; Collins \& Low 2010). Diese Netzwerke können informell (z.B. Familie, Freund/inn/e/n und ethnische Gruppen) oder formell (z. B. Geschäftskontakte, Banken, Anwält/inn/e/n, Regierung, Organisationen und Verbände) sein (Desiderio 2014; Lassalle \& McElwee 2016; KLOOSTERMan et al. 2016).

Studien auf der Grundlage des Mixed-Embeddedness-Ansatzes betonten die Schlüsselrolle von Diaspora-Communities für die Förderung der Entrepreneurship ihrer Mitglieder (KLoOsterman 2003; KNight 2015; Peroni et al. 2016). Die Diasporas sind zugleich informelle soziale Netzwerke, in denen die Immigrant/inn/ en eine gemeinsame Herkunft und kulturelle Identität teilen (McEvoY \& HAFEEZ 2009a). Diese Ähnlichkeiten verstärken die In-Group-Solidarität und Unterstützung, die seinerseits die Entrepreneurship durch die Bereitstellung von Informationen, Unterstützung und praktische Ressourcen fördert (Koelet et al. 2017; Aliaga-Isla \& Rialp 2013). 
Informelle soziale Netzwerke ermöglichen es der Personen in der Diaspora Hindernisse zu überwinden (z. B. fehlende finanzielle Ressourcen), die sie ansonsten an einer erfolgreichen Unternehmensgründung hindern würden. Damit sind diese Netzwerke das wichtigste Instrument im Prozess der Ressourcenmobilisierung (LASSALLE \& McElweE 2016). Diaspora Communities kreieren auch die Nachfrage nach Produkten und Dienstleistungen, die der Kultur und dem Geschmack des Herkunftslandes entsprechen und von Entrepreneurs bedient werden können (McEvoy \& HAfEez 2009b). Diese spezifischen Konsumgewohnheiten ermöglichen es den Entrepreneurs auf Erfahrungen zurückgreifen, die sie in ihren Herkunftsgesellschaften erworben haben. Diaspora Communities wirken wie Magnete auf weitere Immigrant/inn/en. Damit bieten sie eine Quelle für einen Arbeitskräftepool mit Fähigkeiten, die von koethnischen Unternehmer/ inne/n effizienter angezapft werden können als von Unternehmer/inne/n der Aufnahmegesellschaft (SHINNAR \& Young 2008, p. 245).

Nach MARCHAND \& Siegel (2014) sind zugewanderte Frauen mehr auf informelle und formelle Netzwerkunterstützung angewiesen als Männer. Männer weisen stärkere informelle und formelle soziale Bindungen auf. Sie haben damit mehr Zugang zu finanziellen Ressourcen sowie zu allgemeiner Unterstützung für die Gründung und Führung eines Unternehmens. Dieselben argumentieren weiters, dass die größeren Netzwerke von Männern mit Migrationshintergrund vor allem auch aus ihrer stärkeren Präsenz im öffentlichen Raum resultieren. Frauen mit Migrationshintergrund sind mehr im privaten Bereich tätig, weshalb sie öfter Heimarbeitsplätze gründen. Männer mit Migrationshintergrund halten sich mehr in der Öffentlichkeit und im öffentlichen Raum auf, was ihnen gestattet, leichter und effizienter verschiedene Arten von unterstützenden sozialen Netzwerke aufzubauen. Männer in der Diaspora sind meist auch früher migriert als ihre Frauen, haben eine längere Aufenthaltsdauer im Aufnahmeland als die später nachgekommenen Frauen, was den Aufbau von Netzwerken und das Wissen um die Strukturen des Aufnahmelandes erleichtert (ibid.).

\subsubsection{Fazit}

Die theoretischen Konzepte wurden und werden zwar immer differenzierter und unterliegen einer steten Weiterentwicklung, dennoch existiert bis heute kein konsistent ausformuliertes explizierendes Modell, welches die Unternehmertätigkeit von Immigrant/inn/en in allen Facetten zufriedenstellend erklären könnte. Während in der US-amerikanischen Forschung auch rezente Studien zum Thema unter Fokussierung auf die Ressourcen der jeweiligen ethnischen Gruppen und unter nur sehr marginaler Einbeziehung der politisch-institutionellen Rahmenbedingungen durchgeführt werden, ist in den europäischen Studien die Integration der von der Aufnahmegesellschaft dominierten politischen, sozialen und ökonomischen Rahmenbedingungen in die Analyse zum gängigen wissenschaftlichen Standard der Arbeiten zur DiasporaEntrepreneurship geworden. Auch der vorliegende Forschungsbericht orientiert sich an diesem Standard. 